Original Research Paper

\title{
On the Construction and Properties of Lattice-Group Structure in Cartesian Product Spaces
}

\author{
Davronbek Malikov and Susmit Bagchi \\ Department of Aerospace and Software Engineering (Informatics), Gyeongsang National University, Jinju, Republic of Korea
}

\author{
Article history \\ Received: 24-12-2019 \\ Revised: 06-03-2020 \\ Accepted: 3-4-2020 \\ Corresponding Author: \\ Susmit Bagchi \\ Department of Aerospace and \\ Software Engineering \\ (Informatics), Gyeongsang \\ National University, Jinju, \\ Republic of Korea \\ Email: profsbagchi@gmail.com
}

\begin{abstract}
The lattice theory and group algebra have several applications in computing sciences as well as physical sciences. The concept of latticegroup structure is an interesting hybrid algebraic structure having potential applications. In this paper, the algebraic construction of lattice-group structure is formulated and associated algebraic properties are established. The proposed construction considers Cartesian product spaces. The concept of two-dimensional monoid is formulated in Cartesian product spaces of real numbers and a related lattice-group structure is established in the space having reduced dimension. The different categories of functions are employed for dimension reduction while establishing the lattice-group structure. The proposed lattice-monoid and lattice-group structures are finite in nature. The algebraic properties of lattice-group as well as associated structures are formulated. A set of numerical examples are presented in the paper to illustrate structural properties. Finally, the comparative analysis of the proposed structure with other contemporary work is included in the paper.
\end{abstract}

Keywords: Lattice, Group, Lattice-Group, Partial Order, Monoid, Invertibility

\section{Introduction}

Group algebra, especially finite field is the fundamental part of Advanced Encryption Standard (AES). The construction of variants of the DiffieHellman key agreement protocol become easy by using group theory, where the nonAbelian groups can be applied in public key cryptography (Vasco and Steinwandt, 2015; Tzu-Chun, 2018). Furthermore, group theory has applications in physics and particularly in condensed matter physics (Mildred et al., 2010). Moreover, the properties of partial order relation as well as lattice theory are widely applied to various domains of computer science and distributed systems including programming languages (Vijay, 2015). The applications of the lattice theory in distributed computing are comprised of vector clocks design and global predicate detection (Lamport, 1978). The properties of lattice linear predicates enable efficient detection of global predicates in distributed systems (Chase and Garg, 1995). The lattice agreement in asynchronous message passing systems is useful due to its applications in atomic snapshot objects and faulttolerant replicated state machines (Attiya et al., 1995; Xiong et al., 2018). Computational aspects of lattice theory are developed to compute slices for temporal logic formulas (Sen and Garg, 2003). These algorithms are useful in detecting temporal logic formulas in a distributed computation (Mauricio et al., 1999). In other words, partial-order relation and lattices help in obtaining clear, concise and efficient formulations of problems requiring the ability to take transitive closures, solve circular constraints and perform aggregate operations. Lattice theory can be used in the implementation of a knowledge representation language. For example, the knowledge base system is realized by processing a sequence of terminological axioms by using Birkhoff's Representation theorem and finite distributive lattices (Seymour and Marc, 2007; Frank, 2000).

Interestingly, lattice theory plays a role in other branches of mathematics such as, probability theory and graph theory (George, 2009; Louis, 2016). The applications of lattice theory with other results lead to a decomposition technique that expresses all the trees of a graph in the form of set unions of Cartesian products of the sets of subgraphs of the component graphs (Wen-Hai et al., 1990). The algebraic relation between lattice theory and group is an interesting topic having several application possibilities. Thus, the algebraic interrelationship between lattice and groups needs 
attention. The construction of hybrid algebraic structure involving lattice theory and group algebra would be an interesting topic to investigate.

The purpose of this paper is inline to such motivation of formulating algebraic hybrid structures. This paper proposes the construction and algebraic analysis of lattice-group structure in two dimensional Cartesian product spaces of real numbers. It is considered that the lattice-group structure is finite in nature. First we introduce the concept of lattice and lattice-monoid structures in $2 \mathrm{D}$ space. Next, we construct the group structure under the influence of various types of function mappings. The function mappings reduce the dimension while transforming the 2D lattice into a lattice-group structure. The associated algebraic properties of the multidimensional lattices, lattice-monoids and lattice-groups are presented in the paper. The potential applications of the proposed algebraic structure as well as analysis can be made in formulation of model of distributed computing.

The rest of paper is organized as follows. Second section presents preliminary concepts. Third section presents a set of definitions intended to the constructed structures. Fourth section presents a set of analytical properties of the algebraic structures and fifth section presents a set of illustrative examples. The comparative evaluation with the other contemporary works in the domain is presented in sixth section. Finally, seventh section concludes the paper.

\section{Preliminaries}

In this section, we introduce basic definitions and properties related to lattice theory, posets and group algebra.

Binary Relation and Poset (Seymour and Marc, 2007; Thomas, 2004; Dushnik and Miller, 1941; Bernd, 2016)

Let $X$ be a point set and, $A \subset X$ and $B \subset X$ be such that, $A \cap B=\phi$. A binary relation $R$ is an ordered pair such that, $R \subset A \times B$. For any set $A$, a subset of the Cartesian product set $A^{n}$ is called an $n$-ary relation on $A$, where $n \in Z^{+}$. Let $\mathfrak{R}$ be denoting set of real numbers.

A partially ordered set or poset is a set $P$ together with a binary relation $\leq$ such that, the following conditions are satisfied for all $x, y, z \in P$ :

(a) $x \leq x$ (Reflexivity),

(b) If $x \leq y$ and $y \leq x$, then $x=y$ (Anti symmetry),

(c) If $x \leq y$ and $y \leq z$, then $x \leq z$ (Transitivity)

An element $x$ of a poset $P$ is said to be a lower bound for the subset $S \subset P$ if $x \leq s$ for every $s \in S$. The element $x$ is a greatest lower bound of set $S$ if $x$ is a lower bound of $S$ and $y \leq x$ for any lower bound $y$ of set $S$.
Distributive lattice representation (Seymour and Marc, 2007; Birkhoff, 1967; Laszlo, 2015)

An algebraic lattice $(L, \vee, \wedge)$ is a set with two binary operations meet and join $(\wedge, \vee)$ respectively, such that both operations are commutative and associative, where absorption law holds, as mentioned below (where $\forall x, y, z \in X$ ):
(a) $x \wedge y=y \wedge x, x \vee y=y \vee x$,
(b) $x \wedge(y \wedge z)=(x \wedge y) \wedge z$
$x \vee(y \vee z)=(x \vee y) \vee z$,
(c) $x=x \wedge(x \vee y)=x \vee(x \wedge y)$.

A lattice is a partially ordered set in which for every two elements $a$ and $b$ the least upper bound (called join, denoted by, $(a \vee b))$ and the greatest lower bound (called meet, denoted by, $(a \wedge b))$ exist.

A lattice is distributive if it satisfies distributive law given by, $x \wedge(y \vee z)=(x \wedge y) \vee(x \wedge z)$.

Interestingly, a lattice can be represented graphically. The 2D representation of finite partially ordered set $X^{2}$ (with an implied upward orientation) is the directed graph whose vertices are the elements of $X^{2}$ and also, there is a directed edge from $\left(x_{a}, x_{b}\right)$ to $\left(x_{c}, x_{d}\right)$ in the Cartesian product space such that, $\left(x_{a}\right.$, $\left.x_{b}\right) \leq\left(x_{c}, x_{d}\right)$ in $X^{2}$.

The 2D lattice representation is a type of Hasse diagram, which represents the elements of lattice in $X^{2}$. We note that 2D lattice representation of poset $\left(X^{2}, \leq\right)$ need not to be connected.

Binary Operation, Group and Abelian Group (Scott, 1987; Herstein, 1975; Milne, 2013; Robert, 1969)

If $X \neq \phi$ and a binary operation $*$ is defined as $*: X^{2} \rightarrow X$ then the set is called closed under *. A group is given by $G=(X, *)$ such that, the following properties hold:

(a) $\forall x, y, z \in G, x *(y * z)=(x * y) * z$

(b) $\forall x \in G, \exists e \in G: e * x=x * e=x$

(c) $\forall x \in G, \exists x^{-1} \in G: x * x^{-1}=x^{-1} * x=e$

A group $G=\left(X,{ }^{*}\right)$ is called Abelian if it satisfies the commutative law given by, $\forall x, y \in G, x * y=y * x$.

Functions and Invertibility (Seymour and Marc, 2007; William, 2013; Walter, 1976)

A function $f: A \rightarrow B$ is one-to-one if $f(a)=f\left(a^{\prime}\right)$ implies $a=a^{\prime}$. It is said to be an onto function if each element of $B$ is in the image of some element of $A$ 
meaning, $f(A)=B$. A function $f: A \rightarrow B$ is invertible if the function both one-to-one and onto.

\section{Definitions}

In this section, a set of definitions are constructed in relation to multidimensional lattices, lattice-monoids and lattice-group structures. A few of the proposed definitions are direct extension of one-dimensional construction into the multidimensional space.

\section{Relation, Partial Ordering and Quasi Order in 2D}

Let us consider, $\left\{\left(x_{a}, x_{b}\right),\left(x_{c}, x_{d}\right),\left(x_{e}, x_{f}\right), \ldots\left(x_{r}, x_{q}\right)\right.$, $\left.\left(x_{n-1}, x_{n}\right)\right\} \subset X^{2}$ and if it is true that:

$$
\begin{aligned}
& {\left[\left(x_{a}, x_{b}\right) \leq\left(x_{c}, x_{d}\right) \leq \ldots \leq\left(x_{r}, x_{q}\right) \leq\left(x_{n-1}, x_{n}\right)\right] \Rightarrow} \\
& {\left[\left(x_{a} \leq x_{c}\right) \wedge\left(x_{b} \leq x_{d}\right), \ldots \ldots .\left(x_{r} \leq x_{n-1}\right) \wedge\left(x_{q} \leq x_{n}\right)\right]}
\end{aligned}
$$

then $\leq$ is a relation in $X^{2}$.

\section{Remark}

The relation $\leq$ is a partial order relation on $X^{n} \subset \mathfrak{R}^{n}$ if it satisfies following standard properties for $n=2$ :

(a) $\forall\left(x_{a}, x_{a}\right) \in X^{2},\left(x_{a}, x_{a}\right) \in \leq$

(b) If $\left\{\left(x_{a}, x_{b}\right),\left(x_{c}, x_{d}\right)\right\} \subset \leq$ then

$$
\left[\left(x_{a}, x_{b}\right) \leq\left(x_{c}, x_{d}\right)\right] \Leftrightarrow \neg\left[\left(x_{c}, x_{d}\right) \leq\left(x_{a}, x_{b}\right)\right]
$$

and,

(c) If $\left\{\left(x_{a}, x_{b}\right),\left(x_{c}, x_{d}\right),\left(x_{e}, x_{f}\right)\right\} \subset \leq$ then:

$$
\begin{aligned}
& {\left[\left(x_{a}, x_{b}\right) \leq\left(x_{c}, x_{d}\right) \wedge\left(x_{c}, x_{d}\right) \leq\left(x_{e}, x_{f}\right)\right] \Rightarrow} \\
& {\left[\left(x_{a}, x_{b}\right) \leq\left(x_{e}, x_{f}\right)\right] .}
\end{aligned}
$$

We write $\left(X^{2}, \leq\right)$ to specify the poset in Cartesian product space.

Suppose $>$ is a relation on a set $X^{2} \subset \mathfrak{R}^{2}$ satisfying following two properties.

(a) $\forall\left(x_{a}, x_{b}\right) \in X^{2}, \neg\left[\left(x_{a}, x_{b}\right)<\left(x_{a}, x_{b}\right)\right]$

(b) If $\left\{\left(x_{a}, x_{b}\right),\left(x_{c}, x_{d}\right),\left(x_{e}, x_{f}\right)\right\} \subset<$ then

$$
\left[\left(x_{a}, x_{b}\right)<\left(x_{c}, x_{d}\right) \wedge\left(x_{c}, x_{d}\right)<\left(x_{e}, x_{f}\right)\right] \Rightarrow
$$$$
\left[\left(x_{a}, x_{b}\right)<\left(x_{e}, x_{f}\right)\right] \text {. }
$$

In this case, the relation $<$ is called quasi-order on $X^{2}$.

\section{Remark}

Interestingly, there is a close relationship between partial orders and quasi orders. For example, if $\leq$ is a partial order on $X^{2}$, then we can define:

$$
\begin{aligned}
& {\left[\left(x_{a}, x_{b}\right)<\left(x_{c}, x_{d}\right)\right] \Leftrightarrow\left[\left(x_{a}, x_{b}\right) \leq\left(x_{c}, x_{d}\right)\right] \wedge} \\
& {\left[\left(x_{a}, x_{b}\right) \neq\left(x_{c}, x_{d}\right)\right] .}
\end{aligned}
$$

Similarly, if $<$ is quasi order on $X^{2}$, then we can define $\left(x_{a}, x_{b}\right) \leq\left(x_{c}, x_{d}\right)$ as:

$$
\begin{aligned}
& {\left[\left(x_{a}, x_{b}\right) \leq\left(x_{c}, x_{d}\right)\right] \Leftrightarrow\left[\left(x_{a}, x_{b}\right)<\left(x_{c}, x_{d}\right)\right] \vee} \\
& {\left[\left(x_{a}, x_{b}\right)=\left(x_{c}, x_{d}\right)\right] .}
\end{aligned}
$$

Suppose, $\left\{\left(x_{\alpha}, x_{\beta}\right),\left(x_{\gamma}, x_{\mu}\right)\right\} \subset X^{2}$. In this case we say that, if $\left(x_{\alpha}, x_{\beta}\right) \leq\left(x_{\gamma}, x_{\mu}\right)$ and $\left(x_{\gamma}, x_{\mu}\right) \leq\left(x_{\alpha}, x_{\beta}\right)$ then $\left(x_{\alpha}, x_{\beta}\right)$ and $\left(x_{\gamma}, x_{\mu}\right)$ are comparable. If all elements (ordered elements) of $X^{2}$ are comparable, then it said to be totally ordered or linearly ordered (Seymour and Marc, 2007).

\section{Lowest Upper Bound and Greatest Lower Bound in $2 D$}

Let in the algebraic structure in $2 \mathrm{D}$ be $\left\{\left(x_{a}, x_{b}\right),\left(x_{c}\right.\right.$, $\left.\left.x_{d}\right),\left(x_{e}, x_{f}\right),\left(x_{g}, x_{h}\right)\left(x_{l}, x_{m}\right),\left(x_{n}, x_{p}\right)\right\} \subset X^{2}$ and it maintains following condition:

$$
\left(x_{a}, x_{b}\right) \leq\left(x_{c}, x_{d}\right) \leq\left(x_{e}, x_{f}\right) \leq\left(x_{g}, x_{h}\right) \leq\left(x_{l}, x_{m}\right) \leq\left(x_{n}, x_{p}\right) .
$$

In this case:

(a) The supremum of $X^{2}$ is given by:

$$
\sup \left\{\left(x_{a}, x_{b}\right),\left(x_{c}, x_{d}\right),\left(x_{e}, x_{f}\right)\right\}=\left(x_{g}, x_{h}\right)
$$

(b) Accordingly, the infimum of $X^{2}$ is given by:

$$
\inf \left\{\left(x_{g}, x_{h}\right),\left(x_{l}, x_{m}\right),\left(x_{n}, x_{p}\right)\right\}=\left(x_{e}, x_{f}\right) .
$$

\section{Meet and Join in 2D}

Let $\left(X^{2}, \leq\right)$ be a poset. If every pair of elements have sup and inf, then this partial order is called lattice in Cartesian product space and it is denoted by $\left(X^{2}, L, \leq\right)$. The meet and join of $\left(X^{2}, L, \leq\right)$ is given respectively as, $\forall\left(x_{\alpha}, x_{\beta}\right),\left(x_{\gamma}, x_{\mu}\right) \in L$ : 


$$
\left[\left(x_{\alpha}, x_{\beta}\right) \wedge\left(x_{\gamma}, x_{\mu}\right)\right]=\inf \left\{\left(x_{\alpha}, x_{\beta}\right),\left(x_{\gamma}, x_{\mu}\right)\right\}
$$

and,

$$
\left[\left(x_{\alpha}, x_{\beta}\right) \vee\left(x_{\gamma}, x_{\mu}\right)\right]=\sup \left\{\left(x_{\alpha}, x_{\beta}\right),\left(x_{\gamma}, x_{\mu}\right)\right\} .
$$

Any linearly or totally ordered set is a lattice since:

$$
\inf \left\{\left(x_{\alpha}, x_{\beta}\right),\left(x_{\gamma}, x_{\mu}\right)\right\}=\left(x_{\alpha}, x_{\beta}\right)
$$

and,

$$
\sup \left\{\left(x_{\alpha}, x_{\beta}\right),\left(x_{\gamma}, x_{\mu}\right)\right\}=\left(x_{\gamma}, x_{\mu}\right)
$$

whenever $\left(x_{\alpha}, x_{\beta}\right) \leq\left(x_{\gamma}, x_{\mu}\right)$ in $\left(X^{2}, L, \leq\right)$.

\section{Binary Operation in $2 D$}

Let $\left\{\left(x_{a}, x_{b}\right),\left(x_{c}, x_{d}\right)\right\} \subset X^{2}$ be such that, $X^{2} \subset \mathfrak{R}^{2}$ and, $*_{2}: X^{2} \rightarrow X^{2}$ is an algebraic operation in Cartesian product space. If $\left[\left(x_{a}, x_{b}\right) *_{2}\left(x_{c}, x_{d}\right)\right] \Rightarrow\left[\left(x_{\alpha}, x_{\beta}\right) \in X^{2}\right]$, where $x_{\alpha}=\left(x_{a} * x_{c}\right) \in X$ and $x_{\beta}=\left(x_{b} * x_{d}\right) \in X$ then $*_{2}$ is a binary operation on $X^{2}$ having closure property.

\section{Associativity and Identity in $2 D$}

Let $\left\{\left(x_{a}, x_{b}\right),\left(x_{c}, x_{d}\right),\left(x_{e}, x_{f}\right)\right\} \subset X^{2}$ where $X^{2}$ be an arbitrary point set in Cartesian product space. The associativity and identity on $X^{2}$ is defined as:

$$
\begin{aligned}
& \left(x_{a}, x_{b}\right) *_{2}\left[\left(x_{c}, x_{d}\right) *_{2}\left(x_{e}, x_{f}\right)\right] \\
& =\left[\left(x_{a}, x_{b}\right) *_{2}\left(x_{c}, x_{d}\right)\right] *_{2}\left(x_{e}, x_{f}\right),
\end{aligned}
$$

(b) $\forall\left(x_{a}, x_{b}\right) \in X^{2}, \exists(e, e) \in X^{2}$, such that,

$$
\left(x_{a}, x_{b}\right) *_{2}(e, e)=(e, e) *_{2}\left(x_{a}, x_{b}\right)=\left(x_{a}, x_{b}\right) .
$$

The $M=\left(X^{2}, *_{2}\right)$ is called a monoid in Cartesian space. Note that, $M=\left(X^{2}, *_{2}\right)$ is not yet fully equipped with any lattice structure. Moreover, it is important to note that, in our proposed construction, we consider that:

$$
\left(x_{a}, x_{b}\right)^{-1} \neq\left(x_{a}^{-1}, x_{b}^{-1}\right) \text {. }
$$

Before proceeding further, we present a proof that $M$ $=\left(X^{2}, *_{2}\right)$ is indeed a monoid.

\section{Proposition 1}

$M=\left(X^{2}, *_{2}\right)$ is a monoid in 2D.

\section{Proof}

The associativity and identity preservations in algebraic form are given below.

Proof of associativity and identity:

$$
\begin{aligned}
& \left(x_{a}, x_{b}\right) *_{2}\left[\left(x_{c}, x_{d}\right) *_{2}\left(x_{e}, x_{f}\right)\right] \\
& =\left[\left(x_{a}, x_{b}\right) *_{2}\left(x_{c}, x_{d}\right)\right] *_{2}\left(x_{e}, x_{f}\right)=\left(x_{a c e}, x_{b d f}\right) .
\end{aligned}
$$

According to the definition:

$$
\left(x_{a}, x_{b}\right) *_{2}(e, e)=(e, e) *_{2}\left(x_{a}, x_{b}\right)=\left(x_{a} * e, x_{b} * e\right) .
$$

However, inverses do not exist as:

$$
\forall\left(x_{a}, x_{b}\right) \in X^{2},\left(x_{a}, x_{b}\right)^{-1} \neq\left(x_{a}^{-1}, x_{b}^{-1}\right),
$$

thus

$$
\left(x_{a}, x_{b}\right) *_{2}\left(x_{a}, x_{b}\right)^{-1} \neq(e, e) .
$$

Hence, $M=\left(X^{2}, *_{2}\right)$ is indeed a monoid in 2D.

\section{Induced Group and Subgroup}

Let $M=\left(X^{2}, *_{2}\right)$ be a monoid in Cartesian product space. Let a function $f: X^{2} \rightarrow X$ be defined such that, $\forall\left(x_{a}, x_{b}\right) \in X^{2}, \exists x_{\alpha} \in X$ where, $f\left(\left(x_{a}, x_{b}\right)\right)=x_{\alpha}$ then $G_{f}=(M, *, f)$ is called group, if only if, it satisfies standard group axioms under $*: X^{2} \rightarrow X$.

\section{Remark}

We prove associativity of group operation and existence of identity as well as inverse below considering $M=\left(X^{2}, *_{2}\right)$ and its transformation under $f($.$) .$

Let $\left(x_{a}, x_{b}\right),\left(x_{c}, x_{d}\right),\left(x_{e}, x_{f}\right) \in M$ be such that:

(a)

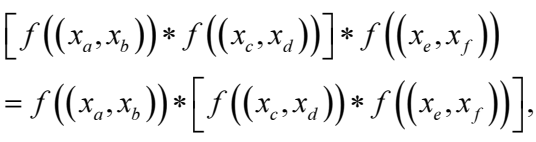

(b) $\exists(e, e) \in M, f((e, e)) * f\left(\left(x_{a}, x_{b}\right)\right)=f\left(\left(x_{a}, x_{b}\right)\right) * f((e, e))$,

(c) $\forall\left(x_{a}, x_{b}\right) \in M, \exists f\left(\left(x_{-a}, x_{-b}\right)\right)$ such that,

$$
f\left(\left(x_{a}, x_{b}\right)\right) * f\left(\left(x_{-a}, x_{-b}\right)\right)=f((e, e)) .
$$

Thus, the algebraic structure $G_{f}=(M, *, f)$ is a group under the influence of $f($.$) , where *: X^{2} \rightarrow X$. If $H_{f} \subset G_{f}$ is a nonempty subset of a group $G_{f}=(M, *, f)$ then, the structure $H_{f}=\left(E,{ }_{H}, f\right)$ is a subgroup of $G_{f}$ iff $H_{f}=\left(E,{ }_{H}{ }_{\text {, }}\right.$, $f)$ maintains all group axioms as mentioned earlier, where $E=\left(Y^{2} \subset X^{2}\right), f: Y^{2} \rightarrow Y$ and $*=*_{H}$. 


\section{Surjective - Homeomorphic Function}

Let $H_{f}$ be a subgroup of $G_{f}$, such that $H_{f}<G_{f}$. The function $f($.) is called as surjective and homeomorphic $(\mathrm{SH})$ if it satisfies following condition:

$$
\forall\left(y_{a}, y_{b}\right),\left(y_{c}, y_{d}\right) \in Y^{2} \subset X^{2}, \exists y_{\alpha} \in Y
$$

such that,

$$
\begin{aligned}
& {\left[f\left(\left(y_{a}, y_{b}\right) *_{2}\left(y_{c}, y_{d}\right)\right)=f\left(\left(y_{a}, y_{b}\right)\right) * f\left(\left(y_{c}, y_{d}\right)\right)\right]} \\
& =\left[y_{\alpha} * y_{\alpha}=y_{2 \alpha} \in Y\right],
\end{aligned}
$$

where, $y_{\alpha}=f\left(\left(y_{a}, y_{b}\right)\right)=f\left(\left(y_{c}, y_{d}\right)\right)$.

\section{Partial Order Monoid in 2D}

Let $\left(X^{2}, \leq\right)$ be a poset and, $M=\left(X^{2},{ }_{2}\right)$ be a monoid. The structure $\left(X^{2}, *_{2}, \leq\right)$ is called a partial-ordermonoid (p-Monoid) if it satisfies following property,

$$
\begin{aligned}
& \forall\left\{\left(x_{a}, x_{b}\right),\left(x_{c}, x_{d}\right),\left(x_{e}, x_{f}\right)\right\} \subset X^{2}, \\
& {\left[\left(x_{a}, x_{b}\right) \leq\left(x_{c}, x_{d}\right)\right] \Rightarrow} \\
& {\left[\left(x_{a}, x_{b}\right) *_{2}\left(x_{e}, x_{f}\right) \leq\left(x_{c}, x_{d}\right) *_{2}\left(x_{e}, x_{f}\right)\right] \wedge} \\
& {\left[\left(x_{e}, x_{f}\right) *_{2}\left(x_{a}, x_{b}\right) \leq\left(x_{e}, x_{f}\right) *_{2}\left(x_{c}, x_{d}\right)\right]}
\end{aligned}
$$

\section{Lattice Monoid in $2 D$}

A p-Monoid $\left(X^{2}, *_{2}, \leq\right)$ is called a lattice-monoid (1Monoid) if and only if it satisfies following conditions:

(a) $\left(X^{2}, \leq\right)$ is a lattice having meet and join,

(b) $\forall\left\{\left(x_{a}, x_{b}\right),\left(x_{c}, x_{d}\right),\left(x_{e}, x_{f}\right)\right\} \subset X^{2}, \nabla \in\{\wedge, \vee\}$,

(b.1) $\begin{aligned} & \left(x_{a}, x_{b}\right) *_{2}\left[\left(x_{c}, x_{d}\right) \nabla\left(x_{e}, x_{f}\right)\right]= \\ & {\left[\left(x_{a}, x_{b}\right) *_{2}\left(x_{c}, x_{d}\right)\right] \nabla\left[\left(x_{a}, x_{b}\right) *_{2}\left(x_{e}, x_{f}\right)\right],}\end{aligned}$ and,
(b.2) $\begin{aligned} & {\left[\left(x_{c}, x_{d}\right) \nabla\left(x_{e}, x_{f}\right)\right] *_{2}\left(x_{a}, x_{b}\right)=} \\ & {\left[\left(x_{c}, x_{d}\right) *_{2}\left(x_{a}, x_{b}\right)\right] \nabla\left[\left(x_{e}, x_{f}\right) *_{2}\left(x_{a}, x_{b}\right)\right] .}\end{aligned}$

The l-Monoid structure is represented by $\left(X^{2}, L,{ }_{2}, \leq\right)$.

\section{Lattice-Group}

Let $G_{f}=(M, *, f)$ be a group under the influence of $f$ : $X^{2} \rightarrow X$. If $\forall x_{\alpha}, x_{\beta} \in f\left(X^{2}\right), x_{\alpha} \wedge x_{\beta}=x_{\alpha}$ and $x_{\alpha} \vee x_{\beta}=x_{\beta}$ then, $L G_{f}=\left(L, G_{f}, f,{ }^{*}, \leq\right)$ is a lattice- group, where $L$ is a lattice on $X$.

\section{Analytical Properties}

In this section, a set of algebraic properties are presented in order to gain insight to the lattice-group and associated structures. First, we consider the strictly positive discrete variety in real $\mathfrak{R}^{n},(n=1,2)$ and investigate whether the group structure can be formed from the monoid in Cartesian product space successfully in the presence of dimension reducing functional map. Accordingly, the set of natural numbers are considered as the domain and codomain of the mapping function, where the domain is a monoid. The resulting behaviour is presented in next theorem.

\section{Theorem 1}

If $G_{f}=\left(M \subseteq N^{2}, \cdot, f\right)$ then $G_{f}$ is not a multiplicative group under surjection $f:\left(X^{2} \subset N^{2}\right) \rightarrow(X \subset N)$, where, $f\left(\left(x_{a}, x_{b}\right)\right)=x_{a}+x_{b} \in N$.

\section{Proof}

Let $G_{f}=\left(M \subseteq N^{2}, \cdot f\right)$, where $M=\left(X^{2} \subseteq N^{2},{ }_{2}\right)$ be a monoid in the Cartesian product space under multiplication, $._{2}: X^{2} \rightarrow X^{2}$. Let $f:\left(X^{2} \subset N^{2}\right) \rightarrow(X \subset N)$ be a surjection where $f\left(\left(x_{a}, x_{b}\right)\right)=x_{a}+x_{b} \in N$. First, we prove that associativity law holds and identity element exists in such settings.

If $\left\{x_{\alpha}, x_{a}, x_{b}, x_{\beta}, x_{c}, x_{d}, x_{\gamma}, x_{e}, x_{f}\right\} \subset N$ such that, $x_{\alpha}=$ $f\left(\left(x_{a}, x_{b}\right)\right), x_{\beta}=f\left(\left(x_{c}, x_{d}\right)\right), x_{\gamma}=f\left(\left(x_{e}, x_{f}\right)\right)$ then $x_{\alpha} .\left(x_{\beta}\right.$. $\left.x_{\gamma}\right)=\left(x_{\alpha} \cdot x_{\beta}\right) \cdot x_{\gamma}$.

If $f($.$) is surjection such that, x_{\alpha}=x_{\beta}$ then $x_{\alpha}{ }^{2} \cdot x_{\gamma}=x_{\gamma} \cdot x_{\alpha}{ }^{2}$, where $x_{\alpha}{ }^{2} \cdot x_{\gamma} \geq 0$.

In this case, the identity element is $\{1\} \subset N$ because, $x_{\alpha} .1=1 . x_{\alpha}=x_{\alpha}$.

However, $\forall x_{i} \in N, x_{i}^{-1} \notin N$, and hence, $G_{f}$ does not have inverse. Thus, $G_{f}=\left(M \subseteq N^{2}, ; f\right)$ is not a multiplicative group. It indicates that, the dimension reducing additive surjection of discrete variety with natural number domain cannot transform a monoid in Cartesian product space into a multiplicative group. However, if the function is not strictly surjective in integer domain, then it can successfully transform a multiplicative group as presented in following example.

Example: Multiplicative Group under Exponential Map

Let there be a real valued function $f\left(\left(x_{a}, x_{b}\right)\right)=e^{x_{a}+x_{b}}$ where $* \equiv \cdot$ (multiplication). It is not considered that $f($.) is surjective.

(a) Associativity:

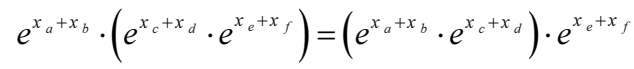

(b) The existence of identity element: $e^{x_{a}+x_{b}} \cdot e^{0}=e^{0} \cdot e^{x_{a}+x_{b}}=e^{x_{a}+x_{b}}$

(c) The existence of inverse: $e^{x_{a}+x_{b}} \cdot e^{-\left(x_{a}+x_{b}\right)}=e^{-\left(x_{a}+x_{b}\right)} \cdot e^{x_{a}+x_{b}}=e^{0}$ 
In this case $G_{f}=(M, \cdot f)$ is a multiplicative group irrespective of surjectivity of $f($.). On the other hand, if the function mapping is surjective in real line, then a centre of Abelian group can be found as illustrated in next theorem. Interestingly, the center has shifting property depending on group operation.

\section{Theorem 2}

If $G_{f}=\left(X^{2} \subseteq \mathfrak{R}^{2},{ }^{*}, f\right)$ is an Abelian group under the surjection $f: \mathfrak{R}^{\overline{2}} \rightarrow \mathfrak{R}$ in Cartesian product space, then there exists a relation-center (R-center) in $\mathfrak{R}$ if $G_{f}=$ $\left(X^{2} \subseteq \mathfrak{R}^{2},+, f\right)$ and, in $\mathfrak{R}^{+}$if $G_{f}=\left(X^{2} \subseteq \mathfrak{R}^{2}, \cdot f\right)$.

\section{Proof}

Let $G_{f}=\left(X^{2} \subseteq \Re^{2}, *, f\right)$ be an algebraic structure in Cartesian product space and $f:\left(X^{2} \subseteq \mathfrak{R}^{2}\right) \rightarrow(X \subseteq \mathfrak{R})$ be a surjection, where $f\left(\left(x_{a}, x_{b}\right)\right)=x_{a} * x_{b} \in \mathfrak{R}$. First, we need to prove that $G_{f}=\left(X^{2} \subseteq \mathfrak{R}^{2}, *, f\right)$ is an Abelian group under the generalized group operation $*: X^{2} \rightarrow X$. Let $\left\{\left(x_{a}, x_{b}\right),\left(x_{c}, x_{d}\right),\left(x_{e}, x_{f}\right)\right\} \subset X^{2}$ and be $\exists x_{\alpha}, x_{\beta}, x_{\gamma} \in X$ such that $x_{\alpha}=f\left(\left(x_{a}, x_{b}\right)\right), x_{\beta}=f\left(\left(x_{c}, x_{d}\right)\right)$, $x_{\gamma}=f\left(\left(x_{e}, x_{f}\right)\right)$. However, according to associativity law, $x_{\alpha} *\left(x_{\alpha} * x_{\alpha}\right)=\left(x_{\alpha} * x_{\alpha}\right) * x_{\alpha} \quad$ as $f($.$) is a surjection$ indicating $x_{\alpha}=x_{\beta}=x_{\gamma}$.

However, if $*=+$ then $\exists 0 \in X$ such that, $x_{\alpha} * 0=x_{\alpha}=0 * x_{\alpha}$. In this case, $\forall x_{\alpha} \in X, \exists\left(-x_{\alpha}\right) \in X$ such that, $x_{\alpha} *\left(-x_{\alpha}\right)=\left(-x_{\alpha}\right) * x_{\alpha}=0$. Moreover, following the commutativity law:

$$
\forall x_{\alpha}, x_{\beta} \in X, x_{\alpha} * x_{\beta}=x_{\beta} * x_{\alpha} .
$$

Hence, $G_{f}=\left(X^{2} \subseteq \mathfrak{R}^{2},+, f\right)$ is an Abelian additive group under surjection $f: \mathfrak{R}^{2} \rightarrow \mathfrak{R}$ in Cartesian product space. Similarly, if $*=\cdot$, then $G_{f}=\left(X^{2} \subseteq \mathfrak{R}^{2}, \cdot f\right)$ is also an Abelian multiplicative group under surjection $f: \mathfrak{R}^{2} \rightarrow \mathfrak{R}$ in Cartesian product space, where identity element is 1 . Thus, the algebraic structure $G_{f}=\left(X^{2} \subseteq \mathfrak{R}^{2},{ }^{*}, f\right)$ is an Abelian group. We will prove the remaining part of the theorem in two parts considering two different group operations as given below.

\section{Case 1: $* \equiv+_{2}$ (Additive Group)}

Let $M=\left(X^{2} \subseteq \mathfrak{R}^{2},+_{2}\right)$ be a monoid in Cartesian product space where $\left(x_{a}, x_{b}\right)+{ }_{2}\left(x_{c}, x_{d}\right)=\left(x_{a}+x_{c}, x_{b}+x_{d}\right)$. Let, $G_{f}$ $=(M,+, f)$ be a group and $H_{f}=\left(E \subset X^{2},+, f\right)$ be a subgroup $H_{f}<G_{f}$ where $f: E \rightarrow(Y \subset X)$ is a surjection. If a relation $R \subset X^{2}$, then $\forall\left(x_{a}, x_{b}\right) \in R, \exists\left(x_{b}, x_{a}\right) \in R^{-1}$ such that, $f\left(\left(x_{a}, x_{b}\right)\right)=f\left(\left(x_{b}, x_{a}\right)\right)=x_{a}+x_{b}$.

Let $\left\{\left(x_{a}, x_{b}\right),\left(x_{b}, x_{a}\right)\right\} \subset\left(R \cup R^{-1}\right)$ be such that, $x_{\alpha} \in H_{f}$ where $f\left(\left(x_{a}, x_{b}\right)\right)=f\left(\left(x_{b}, x_{a}\right)\right)=x_{\alpha}$.
As $H_{f}<G_{f}$, hence $\exists\left\{\left(-x_{a},-x_{b}\right),\left(-x_{b},-x_{a}\right)\right\} \subset\left(R \cup R^{-1}\right)$ such that, $-x_{\alpha} \in H_{f}$ where $f\left(\left(-x_{a},-x_{b}\right)\right)=-f\left(\left(x_{a}, x_{b}\right)\right)$. Thus, $\quad f\left(\left(-x_{a},-x_{b}\right)\right)+f\left(\left(x_{a}, x_{b}\right)\right)=f((0,0))=0 \quad$ and $\{0\} \subset H_{f}, \quad$ where $\quad\{(0,0)\} \subset\left(R \cap R^{-1}\right)$. Hence, $H_{f}=\left\{f\left(\left(x_{a}, x_{b}\right)\right), f((0,0)), f\left(\left(-x_{a},-x_{b}\right)\right)\right\} \subset \mathfrak{R}$ is a R-center of $G_{f}$, under the influence of surjective $f($.).

\section{Case 2: * $\equiv \cdot$ (Multiplicative Group)}

If we replace the group operation with $* \equiv$. indicating multiplicative group, $G_{f}=(M, \cdot f)$ under the surjection $f\left(\left(x_{a}, x_{b}\right)\right)=f\left(\left(x_{b}, x_{a}\right)\right)=x_{a} \cdot x_{b}$, then $\exists\{(1,1)\} \subset\left(R \cap R^{-1}\right)$ and, $f((1,1)) \in H_{f}$ where $H_{f}<G_{f}$. However, if $A \subset\left(R \cup R^{-1}\right)$ indicating, $A \subset \mathfrak{R}^{+} \times \mathfrak{R}^{+}$then $H_{f}=\left\{f\left(\left(x_{a}, x_{b}\right)\right), f((1,1)), f\left(\left(x_{a}^{-1}, x_{b}^{-1}\right)\right)\right\} \subset \mathfrak{R}^{+}$is a $\mathrm{R}-$ center of $G_{f}=(M, \cdot f)$, where $f\left(\left(x_{a}^{-1}, x_{b}^{-1}\right)\right)=\left(x_{a} \cdot x_{b}\right)^{-1} \in \mathfrak{R}^{+}$ (considering $x_{a}>0, x_{b}>0$ ).

It indicates that $H_{f}<G_{f}$ is a relation center (R-center) and it can be shifted on real line based on the nature of group operations.

\section{Examples}

We will present two examples of R-centers of a group based on the nature of group operations.

\section{Example 1}

$\mathrm{R}$-center of additive group.

Let $X \subseteq \Re$ and, $\{(5,4),(-5,-4)\} \subset R \subset X^{2}$. As $H_{f}<$ $G_{f}$, so $\exists\{(4,5),(-4,-5)\} \subset R^{-1} \subset X^{2}$. According to surjectivity, $f((5,4))=f((4,5))=9$ and $f((-5,-4))=f((-4,-5))=-9$. However, $f((5,4))+f((-5$, $-4))=f((0,0))=0$ and thus $\{9,0,-9\}=H_{f}$, which is a Rcenter of group $G_{f}=(M,+, f)$.

\section{Example 2}

R-center of multiplicative group.

Let $\left\{(2,3),\left(-2^{-1},-3^{-1}\right)\right\} \subset R \subset \mathfrak{R}^{2}$. As $H_{f}<G_{f}$, hence $\exists\left\{(3,2),\left(-3^{-1},-2^{-1}\right)\right\} \subset R^{-1} \subset \mathfrak{R}^{2}$.

Following the surjectivity, $f((2,3))=f((3,2))=6 \in \mathfrak{R}^{+}$ and $f\left(\left(-2^{-1},-3^{-1}\right)\right)=f\left(\left(-3^{-1},-2^{-1}\right)\right)=6^{-1} \in \mathfrak{R}^{+}$. So, it is true that, $f((2,3)) \cdot f\left(\left(-2^{-1},-3^{-1}\right)\right)=f((1,1))$. Thus, $H_{f}=\left\{6,0,6^{-1}\right\} \subset \mathfrak{R}^{+}$is a $\mathrm{R}$-center in positive real of Abelian group, $G_{f}=(M, \cdot f)$.

However, if the function $f($.$) is surjective and$ homeomorphic (SH variety), then a cyclic subgroup can be constructed considering a group $G_{f}=\left(X^{2},{ }^{*}, f\right)$ under the abstract algebraic operation having closure in point set $X$. The cyclic subgroup generation requires subset of integer set spanning negative as well as positive ranges 
symmetrically. However, the identity in $2 \mathrm{D}$ should also be mapped through the surjective and homeomorphic function. This property is presented in next theorem.

\section{Theorem 3}

If $f($.$) is surjective and homeomorphic to the$ abstract point set $X$, then $H_{f}=\left(E \subset X^{2},{ }^{*}, f\right)$ is a cyclic group, where $H_{f}<G_{f}=\left(M,{ }^{*}, f\right)$ under abstract algebraic operation, $*: X^{2} \rightarrow X$.

\section{Proof}

Let $X$ be a point set and $G_{f}=\left(M,{ }^{*}, f\right)$ be a group under abstract algebraic operation $*: X^{2} \rightarrow X$ and $H_{f}=$ ( $\left.E \subset X^{2},{ }^{*}, f\right)$ be a subgroup $H_{f}<G_{f}$. Let $\exists A \subset E$ and $\exists B \subset X$. If $\left\{\left(y_{a}, y_{b}\right),\left(y_{c}, y_{d}\right),\left(y_{e}, y_{f}\right)\right\} \subset A$ and $f: A \rightarrow B$ is surjective and homeomorphic then:

$$
f\left(\left(y_{a}, y_{b}\right) *_{2}\left(y_{c}, y_{d}\right)\right)=f\left(y_{a} * y_{c}, y_{b} * y_{d}\right)=f\left(\left(y_{a c}, y_{b d}\right)\right),
$$

and,

$$
\begin{aligned}
& f\left(\left(y_{a c}, y_{b d}\right)\right)=f\left(\left(y_{a}, y_{b}\right)\right) * f\left(\left(y_{c}, y_{d}\right)\right) \\
& =y_{\alpha} * y_{\alpha}=y_{2 \alpha} \in X .
\end{aligned}
$$

It is clear that, according to the surjectivity one can consider,

$$
\begin{aligned}
& f\left(\left(y_{a}, y_{b}\right)\right)=f\left(\left(y_{c}, y_{d}\right)\right)=f\left(\left(y_{e}, y_{f}\right)\right) \\
& =y_{\alpha} \in X .
\end{aligned}
$$

However,

$$
\begin{aligned}
& f\left(\left(y_{c}, y_{d}\right) *_{2}\left(y_{e}, y_{f}\right)\right)=f\left(\left(y_{c} * y_{e}, y_{d} * y_{f}\right)\right) \\
& =f\left(\left(y_{c}, y_{d}\right)\right) * f\left(\left(y_{c}, y_{d}\right)\right)
\end{aligned}
$$

in the algebraic structure under consideration. This indicates,

$$
f\left(\left(y_{c e}, y_{d f}\right)\right)=y_{\alpha} * y_{\alpha}=y_{2 \alpha} \in X
$$

where, $y_{c e}=y_{c} * y_{e}$ and $y_{d f}=y_{d} * y_{f}$.

Moreover,

$$
\begin{aligned}
& f\left(\left(y_{a c}, y_{b d}\right)\right) * f\left(\left(y_{c e}, y_{d f}\right)\right) \\
& =y_{2 \alpha} * y_{2 \alpha}=y_{4 \alpha} \in X .
\end{aligned}
$$

Following the property of associativity,

$$
\begin{aligned}
& f\left(\left(\left(y_{a}, y_{b}\right) *_{2}\left(y_{c}, y_{d}\right)\right) *_{2}\left(y_{e}, y_{f}\right)\right) \\
& =f\left(\left(y_{a} * y_{c}, y_{b} * y_{d}\right) *_{2}\left(y_{e}, y_{f}\right)\right) \\
& =f\left(\left(y_{a c}, y_{b d}\right)\right) * f\left(\left(y_{e}, y_{f}\right)\right)=y_{2 \alpha} * y_{\alpha}=y_{3 \alpha} \in X .
\end{aligned}
$$

However, let $\exists\left\{a_{1}, a_{2}, a_{3} \ldots, a_{n}\right\} \subset X^{2}$, be such that $\left(y_{1}, y_{2}\right)=a_{1},\left(y_{3}, y_{4}\right)=a_{2}, \ldots\left(y_{n-1}, y_{n}\right)=a_{n}$, then:

$$
f\left(a_{1} *_{2} a_{2} *_{2} a_{3} \ldots *_{2} a_{n}\right)=f\left(a_{1} *_{2} a_{2}\right) * f\left(a_{3} *_{2} \ldots *_{2} a_{n}\right) .
$$

However,

$$
f\left(a_{3} *_{2} a_{4} \ldots *_{2} a_{n}\right)=f\left(a_{3} *_{2} a_{4}\right) * f\left(a_{5} *_{2} \ldots . . *_{2} a_{n}\right) .
$$

It indicates,

$$
f\left(a_{1} *_{2} a_{2} *_{2} a_{3} \ldots *_{2} a_{n}\right)=y_{n \beta} \in X
$$

where, $n \in N$ and, $f\left(a_{i} *_{2} a_{i+1}\right)=y_{\beta}$. However, as $G_{f}=$ $\left(M,{ }^{*}, f\right)$ is a group, hence $\exists\left\{\left(y_{1}^{\prime}, y_{2}^{\prime}\right),\left(y_{3}^{\prime}, y_{4}^{\prime}\right), \ldots\left(y_{n-1}^{\prime}, y_{n}^{\prime}\right)\right\} \subset X^{2}$ such that,

$$
f\left(\left(y_{1}^{\prime}, y_{2}^{\prime}\right)\right)=f\left(\left(y_{3}^{\prime}, y_{4}^{\prime}\right)\right)=\ldots=\left(\frac{1}{y_{\beta}}\right) \in X .
$$

It indicates that,

$$
f\left(\left(y_{1}^{\prime}, y_{2}^{\prime}\right) *_{2}\left(y_{3}^{\prime}, y_{4}^{\prime}\right) \ldots *_{2}\left(y_{n-1}^{\prime}, y_{n}^{\prime}\right)=\left(\frac{1}{y_{n \beta}}\right) \in X .\right.
$$

Let $\exists(e, e) \in X^{2}$, be such that $f((e, e))=e \in X$ and, $y_{n \beta} * y_{n \beta}^{-1}=e$. Hence, $H_{f}<G_{f}$ is a cyclic group if $H_{f}=$ $\left(E \subset X^{2},{ }^{*}, f\right)$ and $f(E \backslash\{(e, e)\})=\left\{\left(y_{n \beta}\right)^{i}: n \in V \subset Z, i \in\{1,-\right.$ $1\}\}$, where the identity is, $(e, e) \in E$.

The nature of function mapping affects the resulting group structure. For example, an arbitrary function map may not produce a valid group structure. This property is presented in next theorem. It is important to note that, we have not used any specific definition of function and, we have used generalized classification of two function varieties while constructing the theorem.

\section{Theorem 4}

If $f_{e}: X^{2} \rightarrow X$ is an even function and $f_{o}: X^{2} \rightarrow X$ is an odd function then, algebraic structure $G_{f e}=\left(M,+, f_{e}\right)$ is an additive group and $G_{f o}=\left(M,+, f_{o}\right)$ is not a group in Cartesian product space, where $M=\left(X^{2} \subset \mathfrak{R}^{2},{ }_{2}\right)$.

\section{Proof}

Let $M=\left(X^{2} \subset \mathfrak{R}^{2},{ }_{2}\right)$ be a monoid and $G_{f}=(M,+, f)$ be an algebraic structure in Cartesian product space. Let $\exists A \subset X$ such that $A=\left\{x_{\alpha i}=2 n_{i}, i \in Z^{+}, n_{i} \in Z\right\}$ where $f_{e}: X^{2} \rightarrow A$ is an even function. Let $\exists B \subset X$ be such that $f_{o}: X^{2} \rightarrow B$ is an odd function where $B=\left\{x_{\beta i}=2 n_{i}+1\right.$, $i \in Z^{+}, n_{i} \in Z$. 
Evidently, $f_{e}\left(X^{2}\right) \cap f_{o}\left(X^{2}\right)=\phi, f_{e}\left(X^{2}\right) \cup f_{o}\left(X^{2}\right) \subseteq X$.

Let the algebraic structures generated by $f_{e}($.$) and f_{o}($. be given by, $G_{f e}=\left(M,+, f_{e}\right)$ and $G_{f o}=\left(M,+, f_{o}\right)$ respectively. If $2 n_{i}, 2 n_{j} \in A$, then $2\left(n_{i}+n_{j}\right) \in A$. Let $2 n_{k} \in A$ and by the property of associativity:

$$
2 n_{i}+\left(2 n_{j}+2 n_{k}\right)=\left(2 n_{i}+2 n_{j}\right)+2 n_{k}
$$

Moreover, $\exists f((0,0))=0 \in A$, such that $2 n_{i}+0=0+$ $2 n_{i}, 2 n_{i} \in A$

Given, $2 n_{i} \in A, \exists\left(-2 n_{i}\right) \in A$, such that, $2 n_{i}+\left(-2 n_{i}\right)=$ $\left(-2 n_{i}\right)+2 n_{i}=0 \in A$.

Hence, $G_{f e}=\left(M,+, f_{e}\right)$ is an additive and commutative group under the influence of $f_{e}($.$) .$

Again, let $2 n_{i}+1,2 n_{j}+1 \in B$ and hence $\left(2 n_{i}+1\right)+\left(2 n_{j}+1\right)=2\left(n_{i}+n_{j}+1\right) \notin B$. It indicates that $f_{o}($. is not closed under addition in $G_{f_{o}}$. Hence, $G_{f_{o}}$ is not a group under the influence of $f_{o}($.). Interestingly, the combined varieties of function maps considering surjective-homeomorphic as well as odd and even classifications influence the construction of group structure from the monoid in Cartesian product space. We show that, none of the hybrid varieties of functions successfully constructs a multiplicative group from the monoid structure in Cartesian product space.

\section{Theorem 5}

If $f_{o}($.$) and f_{e}($.$) are surjective-homeomorphic, then$ algebraic structures $G_{f e}=\left(M, \cdot f_{e}\right)$ and $G_{f o}=\left(M, \cdot f_{o}\right)$ can not be groups under multiplication, where $f_{e}: X^{2} \rightarrow X$ is even function and $f_{o}: X^{2} \rightarrow X$ is odd function and $X \subset \mathfrak{R}$.

\section{Proof}

Let $f_{e}: X^{2} \rightarrow X$ be even function such that $\forall\left(y_{a}, y_{b}\right) \in X^{2}, f_{e}\left(\left(y_{a}, y_{b}\right)\right)=2 n_{i} \in X \subset \mathfrak{R}$ where $n_{i} \in Z$. Let $f_{o}: X^{2} \rightarrow X$ be odd function and $\forall\left(y_{a}, y_{b}\right) \in X^{2}$, $f_{o}\left(\left(y_{a}, y_{b}\right)\right)=2 n_{i}+1 \in X \subset \Re$, where $n_{i} \in Z$.

Let two algebraic structures be, $G_{f e}=\left(M, \cdot f_{e}\right)$ and $G_{f o}$ $=\left(M, \cdot f_{o}\right)$, where the monoid in Cartesian product space is given by, $M=\left(X^{2} \subset \mathfrak{R}^{2}, \cdot_{2}\right)$.

Let in the algebraic structure $\left\{\left(y_{a}, y_{b}\right),\left(y_{c}, y_{d}\right),\left(y_{e}, y_{f}\right)\right\}$ $\subset X^{2}$ and $f_{e}($.$) be a SH variety such that$ $\left(x_{a}, x_{b}\right) \cdot_{2}\left(x_{c}, x_{d}\right)=\left(x_{a} \cdot x_{c}, x_{b} \cdot x_{d}\right)$ in $M=\left(X^{2} \subset \mathfrak{R}^{2}, \cdot_{2}\right)$. This indicates, $f_{e}\left(\left(y_{a}, y_{b}\right) \cdot_{2}\left(y_{c}, y_{d}\right)\right)=f_{e}\left(\left(y_{a c}, y_{b d}\right)\right)=4 n_{i}^{2} \in X$, where $y_{a c}=y_{a} \cdot y_{c}$ and $y_{b d}=y_{b} \cdot y_{d}$.
Following the property of associativity under surjective-homeomorphic $f_{e}($.$) ,$

$$
\begin{aligned}
& f_{e}\left(\left(\left(y_{a}, y_{b}\right) \cdot_{2}\left(y_{c}, y_{d}\right)\right)_{2_{2}}\left(y_{e}, y_{f}\right)\right) \\
& =f_{e}\left(\left(y_{a c}, y_{b d}\right)\right) \cdot f_{e}\left(\left(y_{e}, y_{f}\right)\right)=\left(2 n_{i}\right)^{3} \in X \subset \mathfrak{R} .
\end{aligned}
$$

As $M=\left(X^{2}, \cdot_{2}\right)$ is a monoid, hence, $\exists(1,1) \in X^{2}$ in $M=\left(X^{2}, \dot{ }_{2}\right)$. If $G_{f}$ is a group then, $f_{e}((1,1))=1 \in X$ such that the following condition is maintained within the structure:

$$
\begin{aligned}
& \forall\left(y_{a}, y_{b}\right) \in X^{2}, f_{e}\left(\left(y_{a}, y_{b}\right)\right) \cdot f_{e}((1,1))=f_{e}\left(\left(y_{a}, y_{b}\right)\right) \\
& =f_{e}((1,1)) \cdot f_{e}\left(\left(y_{a}, y_{b}\right)\right)
\end{aligned}
$$

indicating the existence of identity in $2 \mathrm{D}$.

However, this leads to a contradiction because $f_{e}((1,1)) \neq 1$. Thus, it is true that, $\forall\left(y_{a}, y_{b}\right) \in X^{2}$, $f_{e}\left(\left(y_{a}, y_{b}\right)\right) \cdot f_{e}((1,1)) \neq f_{e}\left(\left(y_{a}, y_{b}\right)\right)$.

Hence, $G_{f e}=\left(M, \cdot, f_{e}\right)$ is not a group.

On the other hand, if $f_{o}($.$) is a surjective and$ homeomorphic function then:

$$
f_{o}\left(\left(y_{a}, y_{b}\right) \cdot_{2}\left(y_{c}, y_{d}\right)\right)=f_{o}\left(\left(y_{a c}, y_{b d}\right)\right) \text {, }
$$

and furthermore:

$$
\begin{aligned}
& f_{o}\left(\left(y_{a c}, y_{b d}\right)\right)=f_{o}\left(\left(y_{a}, y_{b}\right)\right) \cdot f_{o}\left(\left(y_{c}, y_{d}\right)\right) \\
& =2\left(2 n_{i}^{2}+2 n_{i}\right)+1
\end{aligned}
$$

where $y_{a c}=y_{a} \cdot y_{c}$ and $y_{b d}=y_{b} \cdot y_{d}$.

Following the property of associativity under surjective-homeomorphic function $f_{o}($.$) :$

$$
\begin{aligned}
& f_{o}\left(\left(\left(y_{a}, y_{b}\right) \cdot_{2}\left(y_{c}, y_{d}\right)\right) \cdot_{2}\left(y_{e}, y_{f}\right)\right) \\
& =f_{o}\left(\left(y_{a c}, y_{b d}\right)\right) \cdot f_{o}\left(\left(y_{e}, y_{f}\right)\right) \\
& =2\left(4 n_{i}^{3}+6 n_{i}^{2}+3 n_{i}+1\right) \in X \subset \Re .
\end{aligned}
$$

As $M=\left(X^{2}, \cdot_{2}\right)$ is a monoid, so $\exists(1,1) \in X^{2}$ in $M=\left(X^{2},{ }_{2}\right)$ and, $\forall\left(y_{a}, y_{b}\right) \in X^{2}$ :

$$
\begin{aligned}
& f_{o}\left(\left(y_{a}, y_{b}\right)\right) \cdot f_{o}((1,1)) \\
& =f_{o}((1,1)) \cdot f_{o}\left(\left(y_{a}, y_{b}\right)\right)=f_{o}\left(\left(y_{a}, y_{b}\right)\right) .
\end{aligned}
$$

Hence, $f_{o}((1,1))=1 \in X$, which satisfies the required condition for odd function. Furthermore, if $G_{f o}$ is a group, then given: 


$$
\begin{aligned}
& f_{o}\left(\left(y_{a}, y_{b}\right)\right)=\left(2 n_{i}+1\right) \in X \subset \Re, \\
& \exists f_{o}\left(\left(y_{a}^{\prime}, y_{b}^{\prime}\right)\right)=\left(2 n_{i}+1\right)^{-1} \in X \subset \Re,
\end{aligned}
$$

where $\left(y_{a}^{\prime}, y_{b}^{\prime}\right) \in X^{2}$ such that,

$$
\begin{aligned}
& f_{o}\left(\left(y_{a}, y_{b}\right)\right) \cdot f_{o}\left(\left(y_{a}^{\prime}, y_{b}^{\prime}\right)\right) \\
& =f_{o}\left(\left(y_{a}^{\prime}, y_{b}^{\prime}\right)\right) \cdot f_{o}\left(\left(y_{a}, y_{b}\right)\right)=f_{o}((1,1)) .
\end{aligned}
$$

However, as, $f_{o}($.$) is odd function, hence$ $f_{o}\left(\left(y_{a}^{\prime}, y_{b}^{\prime}\right)\right) \neq\left(2 n_{i}+1\right)^{-1}$.

Thus, $\forall\left(y_{a}, y_{b}\right),\left(y_{a}^{\prime}, y_{b}^{\prime}\right) \in X^{2}$,

$$
f_{o}\left(\left(y_{a}, y_{b}\right)\right) \cdot f_{o}\left(\left(y_{a}^{\prime}, y_{b}^{\prime}\right)\right) \neq f_{o}((1,1)) \text {. }
$$

Hence, $G_{f o}=\left(M, \cdot f_{o}\right)$ is not a group.

The algebraic structure can be further enriched by incorporating the partial ordering relation in the Cartesian product space. We introduce the lattice structure into the partially ordered monoid to form a hybrid lattice-monoid structure in 2D. In the next theorem we illustrate that the 2-dimensional latticemonoid is easy to formulate considering whole real line $\mathfrak{R}$ generating combinatorial forms of ordered pairs in Cartesian product space.

\section{Theorem 6}

Let $\left(X^{2},{ }_{2}, \leq\right)$ be a partial order monoid. If $* \equiv+$, then, there exists lattice-monoid $\left(X^{2}, L,+_{2}, \leq\right)$ in Cartesian product space, where $+_{2}: X^{2} \rightarrow X^{2}$ and $+: X^{2} \rightarrow X$.

\section{Proof}

Let $\left(X^{2}, *_{2}, \leq\right)$ be a partial order monoid in Cartesian product space, where $\leq$ is a partially ordered relation in 2D. First, we will consider the case where elements are negative reals as, $\left\{x_{a}, x_{b}\right\} \subset \mathfrak{R} \backslash \mathfrak{R}^{+}$and $\left\{\left(x_{c}, x_{d}\right),\left(x_{e}, x_{f}\right)\right\}$ $\subset\left(\Re^{+}\right)^{2}$. If $*=+_{2}$ then by following the definition of lattice-monoid, one can derive as follows:

$$
\begin{aligned}
& \left\{\left(x_{a}, x_{b}\right),\left(x_{c}, x_{d}\right),\left(x_{e}, x_{f}\right)\right\} \subset\left(X^{2}, L, \leq\right), \\
& \left(x_{a}, x_{b}\right)+{ }_{2}\left[\left(x_{c}, x_{d}\right) \vee\left(x_{e}, x_{f}\right)\right]=\left(x_{a}, x_{b}\right)+{ }_{2}\left(x_{e}, x_{f}\right), \\
& \left(x_{a}, x_{b}\right)++_{2}\left(x_{e}, x_{f}\right)=\left(x_{a}+x_{e}, x_{b}+x_{f}\right)
\end{aligned}
$$

Furthermore, in $\left(X^{2}, L, \leq\right)$ the following equality holds:

$$
\begin{aligned}
& {\left[\left(x_{a}, x_{b}\right)++_{2}\left(x_{c}, x_{d}\right)\right] \vee\left[\left(x_{a}, x_{b}\right)++_{2}\left(x_{e}, x_{f}\right)\right]} \\
& =\left[\left(x_{a}+x_{c}, x_{b}+x_{d}\right)\right] \vee\left[\left(x_{a}+x_{e}, x_{b}+x_{f}\right)\right] \\
& =\left[\left(x_{a}+x_{e}, x_{b}+x_{f}\right)\right]
\end{aligned}
$$

Thus, the equations (16) and (17) lead to:

$$
\begin{aligned}
& \left(x_{a}, x_{b}\right)+_{2}\left[\left(x_{c}, x_{d}\right) \vee\left(x_{e}, x_{f}\right)\right] \\
& =\left[\left(x_{a}+x_{c}, x_{b}+x_{d}\right)\right] \vee\left[\left(x_{a}+x_{e}, x_{b}+x_{f}\right)\right] .
\end{aligned}
$$

Again, in another case let us consider that $\left(x_{a}, x_{b}\right) \in$ $X^{2}$ such that $x_{a} \in \mathfrak{R} \backslash \mathfrak{R}^{+}$and $x_{b} \in \mathfrak{R}^{+}$and $\left\{\left(x_{c}, x_{d}\right)\right.$, $\left.\left(x_{e}, x_{f}\right)\right\} \subset\left(\mathfrak{R}^{+}\right)^{2}$. By following the lattice-monoid definition one can conclude that:

$$
\left(x_{a}, x_{b}\right)++_{2}\left[\left(x_{c}, x_{d}\right) \vee\left(x_{e}, x_{f}\right)\right]=\left(x_{a}+x_{e}, x_{b}+x_{f}\right)
$$

and,

$$
\begin{aligned}
& {\left[\left(x_{a}, x_{b}\right)++_{2}\left(x_{c}, x_{d}\right)\right] \vee\left[\left(x_{a}, x_{b}\right)++_{2}\left(x_{e}, x_{f}\right)\right]} \\
& =\left(x_{a}+x_{e}, x_{b}+x_{f}\right)
\end{aligned}
$$

Hence,

$$
\begin{aligned}
& \left(x_{a}, x_{b}\right)++_{2}\left[\left(x_{c}, x_{d}\right) \vee\left(x_{e}, x_{f}\right)\right] \\
& =\left[\left(x_{a}, x_{b}\right)++_{2}\left(x_{c}, x_{d}\right)\right] \vee\left[\left(x_{a}, x_{b}\right)+{ }_{2}\left(x_{e}, x_{f}\right)\right]
\end{aligned}
$$

if $x_{a} \in \mathfrak{R} \backslash \mathfrak{R}^{+}, \quad x_{b} \in \mathfrak{R}^{+}$and $\left\{\left(x_{c}, x_{d}\right),\left(x_{e}, x_{f}\right)\right\} \subset\left(\mathfrak{R}^{+}\right)^{2}$.

Likewise, if $x_{a} \in \mathfrak{R}^{+}, x_{b} \in \mathfrak{R} \backslash \mathfrak{R}^{+}$and $\left\{\left(x_{c}, x_{d}\right),\left(x_{e}\right.\right.$, $\left.\left.x_{f}\right)\right\} \subset\left(\Re^{+}\right)^{2}$ then also the lattice-monoid structural property is maintained. Hence, there exists latticemonoid $\left(X^{2}, L,+_{2}, \leq\right)$ in Cartesian product space in all cases. However, if the lattice-monoid is a multiplicative variety and the partially ordered elements are not strictly in positive real plane, then the lattice-monoid structure fails to exist. The observation is presented in next lemma.

\section{Lemma 6.1}

In $\left(X^{2}, \dot{ }_{2}, \leq\right)$ partially ordered monoid, if $\left\{\left(x_{a}, x_{b}\right),\left(x_{c}, x_{d}\right),\left(x_{e}, x_{f}\right)\right\} \subset X^{2}, \exists\left(x_{a}, x_{b}\right) \in \mathfrak{R}^{2} \backslash\left(\mathfrak{R}^{+}\right)^{2}$, then $\left(X^{2}, L,{ }_{2}, \leq\right)$ does not exist in Cartesian product space.

\section{Proof}

Let $\left(X^{2},{ }_{2}, \leq\right)$ be a partially order monoid in $2 \mathrm{D}$, where $X^{2} \subset \mathfrak{R}^{2}$ and $\cdot_{2}: X^{2} \rightarrow X^{2}$ be such that $\left(x_{a}, x_{b}\right) \cdot_{2}\left(x_{c}, x_{d}\right)=\left(x_{a} \cdot x_{c}, x_{b} \cdot x_{d}\right)$. Let $\leq$ be a partial order 
relation in 2D. Let $\left\{x_{a}, x_{b}\right\} \subset \mathfrak{R} \backslash \mathfrak{R}^{+} \quad$ and, let $\left\{\left(x_{c}, x_{d}\right),\left(x_{e}, x_{f}\right)\right\} \subset\left(\mathfrak{R}^{+}\right)^{2}$.

According to the definition of lattice-monoid, the following relation is established considering $\left(x_{c}, x_{d}\right) \leq\left(x_{e}, x_{f}\right)$ :

$$
\left(x_{a}, x_{b}\right) \cdot \cdot_{2}\left[\left(x_{c}, x_{d}\right) \vee\left(x_{e}, x_{f}\right)\right]=\left(x_{a e}, x_{b f}\right)
$$

Moreover, the following algebraic relation can be established:

$$
\left[\left(x_{a}, x_{b}\right) \cdot_{2}\left(x_{c}, x_{d}\right)\right] \vee\left[\left(x_{a}, x_{b}\right) \cdot_{2}\left(x_{e}, x_{f}\right)\right]=\left(x_{a c}, x_{b d}\right)
$$

where, $x_{a c}=x_{a} \cdot x_{c}, x_{a e}=x_{a} \cdot x_{e}, x_{b d}=x_{b} \cdot x_{d}$ and $x_{b f}=x_{b} \cdot x_{f}$.

Hence, in Cartesian product space:

$$
\begin{aligned}
& \left(x_{a}, x_{b}\right) \cdot \cdot_{2}\left[\left(x_{c}, x_{d}\right) \vee\left(x_{e}, x_{f}\right)\right] \\
& \neq\left[\left(x_{a}, x_{b}\right) \cdot{ }_{2}\left(x_{c}, x_{d}\right)\right] \vee\left[\left(x_{a}, x_{b}\right) \cdot{ }_{2}\left(x_{e}, x_{f}\right)\right] .
\end{aligned}
$$

However, let $\left(x_{a}, x_{b}\right) \in X^{2}$ be such that $x_{a} \in \mathfrak{R} \backslash \mathfrak{R}^{+}$, $x_{b} \in \mathfrak{R}^{+}$and $\left\{\left(x_{c}, x_{d}\right),\left(x_{e}, x_{f}\right)\right\} \subset\left(\mathfrak{R}^{+}\right)^{2}$. According to the definition of lattice-monoid as mentioned earlier:

$$
\left(x_{a}, x_{b}\right) \cdot \cdot_{2}\left[\left(x_{c}, x_{d}\right) \vee\left(x_{e}, x_{f}\right)\right]=\left(x_{a e}, x_{b f}\right)
$$

Again, it can be verified that,

$$
\begin{aligned}
& {\left[\left(x_{a}, x_{b}\right) \cdot_{2}\left(x_{c}, x_{d}\right)\right] \vee\left[\left(x_{a}, x_{b}\right)_{2}\left(x_{e}, x_{f}\right)\right]} \\
& =\left[\left(x_{a c}, x_{b d}\right) \vee\left(x_{a e}, x_{b f}\right)\right]
\end{aligned}
$$

However, $\left[\left(x_{a c}, x_{b d}\right) \vee\left(x_{a e}, x_{b f}\right)\right] \neq\left(x_{a e}, x_{b f}\right)$ since $\left\{\left(x_{a c}, x_{b d}\right),\left(x_{a e}, x_{b f}\right)\right\} \not \subset \leq$. Furthermore, let $x_{a} \in \mathfrak{R}^{+}$, $x_{b} \in \mathfrak{R} \backslash \mathfrak{R}^{+}$be in $X$. According to the lattice-monoid definition,

$$
\begin{aligned}
& \left(x_{a}, x_{b}\right) \cdot_{2}\left[\left(x_{c}, x_{d}\right) \vee\left(x_{e}, x_{f}\right)\right]=\left(x_{a e}, x_{b f}\right) \text { and, } \\
& {\left[\left(x_{a}, x_{b}\right) \cdot_{2}\left(x_{c}, x_{d}\right)\right] \vee\left[\left(x_{a}, x_{b}\right) \cdot_{2}\left(x_{e}, x_{f}\right)\right]} \\
& =\left[\left(x_{a c}, x_{b d}\right) \vee\left(x_{a e}, x_{b f}\right)\right]
\end{aligned}
$$

Similarly, in this case also, $\left[\left(x_{a c}, x_{b d}\right) \vee\left(x_{a e}, x_{b f}\right)\right]$ $\neq\left(x_{a e}, x_{b f}\right)$. Hence, $\left(X^{2},{ }_{2}, \leq\right)$ is not a lattice-monoid in Cartesian product space under multiplication operation except the case, where $\left(x_{a}, x_{b}\right) \in\left(\mathfrak{R}^{+}\right)^{2}$ and, $\left\{\left(x_{c}, x_{d}\right),\left(x_{e}, x_{f}\right)\right\} \subset\left(\mathfrak{R}^{+}\right)^{2}$. The interesting question is whether the lattice-monoid can retain the distributive property and, if so then what the required condition is. This property is analyzed in next theorem. We show that, every lattice-monoid is distributive in nature, if exists.

\section{Theorem 7}

Every lattice-monoid $\left(X^{2}, L, *, \leq\right)$ is distributive in Cartesian product space, if exists.

\section{Proof}

Let $X$ be a point set and $\left(X^{2}, L, *_{2}, \leq\right)$ be a latticemonoid in Cartesian product space, where $*_{2}: X^{2} \rightarrow X^{2}$ and $*: X^{2} \rightarrow X$ are abstract algebraic operations and $\leq$ is a partial order relation in $2 \mathrm{D}$.

Let us consider the elements as, $\left\{\left(x_{a}, x_{b}\right),\left(x_{c}, x_{d}\right),\left(x_{e}, x_{f}\right)\right\}$ $\subset X^{2}$. As $\left(X^{2}, L, *_{2}, \leq\right)$ is a lattice-monoid, hence $\forall\left(x_{a}, x_{b}\right),\left(x_{c}, x_{d}\right) \in X^{2}$, there exists join and meet in $2 \mathrm{D}$. However, as, $\leq$ is a relation in $2 \mathrm{D}$, hence:

$$
\left[\left(x_{a}, x_{b}\right) \leq\left(x_{c}, x_{d}\right)\right] \Rightarrow\left[\left(x_{a} \leq x_{c}\right) \wedge\left(x_{b} \leq x_{d}\right)\right] .
$$

Moreover, following the distributive law it can be derived as:

$$
\left(x_{a}, x_{b}\right) \wedge\left[\left(x_{c}, x_{d}\right) \vee\left(x_{e}, x_{f}\right)\right]=\left(x_{a}, x_{b}\right)
$$

Furthermore, the following equation is maintained in the lattice-monoid under partial order,

$$
\left[\left(x_{a}, x_{b}\right) \wedge\left(x_{c}, x_{d}\right)\right] \vee\left[\left(x_{a}, x_{b}\right) \wedge\left(x_{e}, x_{f}\right)\right]=\left(x_{a}, x_{b}\right)
$$

Hence, it is verified that:

$$
\left(x_{a}, x_{b}\right) \wedge\left[\left(x_{c}, x_{d}\right) \vee\left(x_{e}, x_{f}\right)\right]=\left(x_{a}, x_{b}\right) .
$$

However, one can verify that distributive property holds under lattice join operation as given below:

$$
\left(x_{a}, x_{b}\right) \vee\left[\left(x_{c}, x_{d}\right) \wedge\left(x_{e}, x_{f}\right)\right]=\left(x_{c}, x_{d}\right),
$$

and,

$$
\left[\left(x_{a}, x_{b}\right) \vee\left(x_{c}, x_{d}\right)\right] \wedge\left[\left(x_{a}, x_{b}\right) \vee\left(x_{e}, x_{f}\right)\right]=\left(x_{c}, x_{d}\right) .
$$

Hence, every lattice-monoid $\left(X^{2}, L, *_{2}, \leq\right)$ is distributive in Cartesian product space.

There is an interplay between lattice-group, arbitrary function mapping and distributive property. It is possible 
to construct a lattice-subgroup considering an arbitrary function mapping having domain in $2 \mathrm{D}$. We illustrate the interaction between lattice-group and an arbitrary function in the next theorem.

\section{Theorem 8}

In $G_{f}=(M, *, f)$ if $f:(Y \subset X)^{2} \rightarrow(Y \subset X)$ is any arbitrary function, then $L H_{f}=\left(L, H_{f}, f, *\right)$ is a distributive lattice-subgroup, where $H_{f}<G_{f}$.

\section{Proof}

We will prove this theorem in three parts considering variations of mapping such as surjective, injective and bijective $f($.).

Let $G_{f}=(M, *, f)$ be a group under the influence of $f\left(\right.$.) . Let $H_{f}=(E, *, f)$ be a subgroup $\left(H_{f}<G_{f}\right)$ where, $E=Y^{2} \subset X^{2}$ and $f:(Y \subset X)^{2} \rightarrow(Y \subset X)$ be any arbitrary function.

\section{Case 1: $f($.$) is Surjective$}

As, $G_{f}=(M, *, f)$ is a group, hence it is true that, $\forall\left(y_{a}, y_{b}\right),\left(y_{c}, y_{d}\right),\left(y_{e}, y_{f}\right) \in Y^{2}, Y \subset X, \exists y_{\alpha}, y_{\beta}, y_{\gamma} \in Y$, where $f\left(\left(y_{a}, y_{b}\right)\right)=y_{\alpha}, f\left(\left(y_{c}, y_{d}\right)\right)=y_{\beta}, f\left(\left(y_{e}, y_{f}\right)\right)=y_{\gamma} \quad$ and $\left\{y_{\alpha}, y_{\beta}, y_{\gamma}\right\}$ is a subset of $G_{f}$.

However, if $f:(Y \subset X)^{2} \rightarrow(Y \subset X)$ is surjective then:

$$
f\left(\left(y_{a}, y_{b}\right)\right)=f\left(\left(y_{c}, y_{d}\right)\right)=f\left(\left(y_{e}, y_{f}\right)\right)=y_{\alpha} .
$$

Thus, by following the group axioms it can be concluded that:

$$
y_{\alpha} *\left(y_{\alpha} * y_{\alpha}\right)=\left(y_{\alpha} * y_{\alpha}\right) * y_{\alpha}
$$

However, as $M$ is a monoid so, $\exists(e, e) \in Y^{2}, \exists e \in X$, such that $f((e, e))=e$ and:

$$
y_{\alpha} * e=e * y_{\alpha}=y_{\alpha}
$$

Hence, $H_{f}<G_{f}$ if $\{e\} \subset H_{f}$ and $\forall f\left(\left(y_{1}, y_{2}\right)\right) \in Y$, $\exists f\left(\left(y_{1}^{\prime}, y_{2}^{\prime}\right)\right) \in Y \quad$ such that, $f\left(\left(y_{1}, y_{2}\right)\right) * f\left(\left(y_{1}^{\prime}, y_{2}^{\prime}\right)\right)$ $=f((e, e))$. Moreover, if $f\left(\left(y_{1}, y_{2}\right)\right)$ is in $H_{f}$ then it indicates that $f\left(\left(y_{1}^{\prime}, y_{2}^{\prime}\right)\right)$ is also in $H_{f}$.

This is to note that, we are not enforcing any surjectivity under relational symmetry $f\left(\left(y_{1}, y_{2}\right)\right)=f\left(\left(y_{2}, y_{1}\right)\right)$ maintaining generality.

Hence, $H_{f}=(E, *, f)$ is a subgroup of $G_{f}=(M, *, f)$ under the influence of surjective map without the requirement of symmetric relation. Moreover, according to the distributive law:

$$
y_{\alpha} \wedge\left(y_{\alpha} \vee y_{\alpha}\right)=\left(y_{\alpha} \wedge y_{\alpha}\right) \vee\left(y_{\alpha} \wedge y_{\alpha}\right)
$$

Similarly for $y_{\beta}, y_{\gamma} \in f($.$) the distributive law holds.$ It indicates that $L H_{f}=\left(L, H_{f}, f, *\right)$ is a distributive lattice-subgroup where $f($.) is surjective.

\section{Case 2: $f($.) is Injective}

Let $f:(Y \subset X)^{2} \rightarrow(Y \subset X)$ be injective such that $\left\{y_{\alpha}, y_{\beta}, y_{\gamma}\right\} \subset f\left(Y^{2}\right)$ and, $y_{\alpha} \neq y_{\beta} \neq y_{\gamma}$ where, $\left\{y_{\alpha}, y_{\beta}, y_{\gamma}\right\}$ is a subset of $G_{f}$. According to the associativity property of groups with respect to algebraic operation $*: X^{2} \rightarrow X$ :

$$
y_{\alpha} *\left(y_{\beta} * y_{\gamma}\right)=\left(y_{\alpha} * y_{\beta}\right) * y_{\gamma}
$$

In the monoid in Cartesian product space, $M=\left(X^{2}, *_{2}\right), \exists(e, e) \in X^{2}$ such that:

$$
\begin{aligned}
& f\left(\left(y_{1}, y_{2}\right)\right)=f((e, e)) * f\left(\left(y_{1}, y_{2}\right)\right) \\
& =f\left(\left(y_{1}, y_{2}\right)\right) * f((e, e))
\end{aligned}
$$

and,

$$
\forall f\left(\left(y_{1}, y_{2}\right)\right) \in Y, \exists f\left(\left(y_{1}^{\prime}, y_{2}^{\prime}\right)\right) \in Y
$$

such that,

$$
f\left(\left(y_{1}, y_{2}\right)\right) * f\left(\left(y_{1}^{\prime}, y_{2}^{\prime}\right)\right)=f((e, e))
$$

indicating $G_{f}$ is a group under $f($.$) .$

Hence, if $f\left(\left(y_{1}^{\prime}, y_{2}^{\prime}\right)\right)$ is in $G_{f}$ then, $f\left(\left(y_{1}, y_{2}\right)\right) \neq f\left(\left(y_{1}^{\prime}, y_{2}^{\prime}\right)\right)$ maintaining injectivity of $f($.$) .$

Hence, if $H_{f}=(E \subset Y, *, f)$ where, the algebraic operation $*$ is closed in $E$ and $H_{f}$ maintains other group axioms including $f((e, e)) \in E$, then $H_{f}=(E, *, f)$ is a subgroup of $G_{f}=(M, *, f)$. Furthermore, according to the distributive law, $y_{\alpha} \wedge\left(y_{\beta} \vee y_{\gamma}\right)=\left(y_{\alpha} \wedge y_{\beta}\right) \vee\left(y_{\alpha} \wedge y_{\gamma}\right)$ because $y_{\alpha} \leq y_{\beta} \leq y_{\gamma}$.

Thus, $L H_{f}=\left(L, H_{f}, f, *\right)$ is a distributive latticesubgroup where $f($.$) is injective.$

\section{Case 3: $f($.$) is Bijective$}

Let $f:(Y \subset X)^{2} \rightarrow(Y \subset X)$ be a bijective map and, $G_{f}=(M, *, f)$ be a group. If $E \subset Y$ such that, 
$\forall f\left(\left(y_{1}, y_{2}\right)\right) \in E, \quad \exists f((e, e)) \in E$ where, $f\left(\left(y_{1}, y_{2}\right)\right) * f((e, e))$ $=f\left(\left(y_{1}, y_{2}\right)\right)$ then, $(E, *)$ is a monoid in 1D. Moreover, $\forall f\left(\left(y_{1}, y_{2}\right)\right) \in E, \quad \exists f\left(\left(y_{1}^{\prime}, y_{2}^{\prime}\right)\right) \in E$ such that:

$$
\begin{aligned}
& f\left(\left(y_{1}, y_{2}\right)\right) * f\left(\left(y_{1}^{\prime}, y_{2}^{\prime}\right)\right) \\
& =f\left(\left(y_{1}^{\prime}, y_{2}^{\prime}\right)\right) * f\left(\left(y_{1}, y_{2}\right)\right)=f((e, e))
\end{aligned}
$$

and, $H_{f}<G_{f}$

where, $H_{f}=(E, *, f)$ considering * is closed in $E$. Again, as $f\left(\right.$.) is bijective, hence, $\forall y_{\alpha}, y_{\beta} \in E, \quad y_{\alpha} \neq y_{\beta}$. Furthermore, by following the distributive lattice property if $y_{\alpha} \leq y_{\beta} \leq y_{\gamma}$ then, $y_{\alpha} \wedge\left(y_{\beta} \vee y_{\gamma}\right)=\left(y_{\alpha} \wedge y_{\beta}\right) \vee\left(y_{\alpha} \wedge y_{\gamma}\right)$.

Hence, $H_{f}<G_{f}$ and $L H_{f}$ is a distributive latticesubgroup. Thus, $L H_{f}=\left(L, H_{f}, f, *\right)$ is a distributive lattice-subgroup independent of the nature of function mapping. This indicates that, formation of distributive lattice-subgroup structure is possible irrespective of invertibility of the mapping from Cartesian product space to 1D. However, the characteristics of such mapping are important to maintain the resulting lattice-group structure, $L G_{f}=\left(L, G_{f}, f, *\right)$.

The monotonicity of the function plays an important role in establishing the lattice-group structure as indicated in next theorem.

\section{Theorem 9}

If $f($.$) is monotonically decreasing function in group$ $G_{f}=(M, *, f)$, then $L G_{f}=\left(L, G_{f}, f, *, \leq\right)$ is not a latticegroup.

\section{Proof}

Let $X$ be a point set and $G_{f}=\left(M,{ }^{*}, f\right)$ be a group, where $*: X^{2} \rightarrow X$ is an abstract algebraic operation and $\leq$ is a partial order relation in Cartesian product space. Let $f: X^{2} \rightarrow X$ be a monotonically decreasing function such that:

$$
\begin{aligned}
& \forall\left\{\left(x_{a}, x_{b}\right),\left(x_{c}, x_{d}\right),\left(x_{e}, x_{f}\right)\right\} \subset X^{2}, \\
& f\left(\left(x_{a}, x_{b}\right)\right) \geq f\left(\left(x_{c}, x_{d}\right)\right) \geq f\left(\left(x_{e}, x_{f}\right)\right)
\end{aligned}
$$

where, $\left(x_{a}, x_{b}\right) \leq\left(x_{c}, x_{d}\right) \leq\left(x_{e}, x_{f}\right)$.

However, according to the lattice-group definition, $\forall x_{\alpha}, x_{\beta} \in f\left(X^{2}\right), \quad x_{\alpha} \wedge x_{\beta}=x_{\alpha} \quad$ and $\quad x_{\alpha} \vee x_{\beta}=x_{\beta}$, where $f\left(\left(x_{a}, x_{b}\right)\right)=x_{\alpha}$ and, $f\left(\left(x_{c}, x_{d}\right)\right)=x_{\beta}$. In this case, as $x_{\alpha} \geq x_{\beta} \geq x_{\gamma}$ due to monotonically decreasing function, hence $x_{\alpha} \wedge x_{\beta} \neq x_{\alpha}$ and $x_{\alpha} \vee x_{\beta} \neq x_{\beta}$.

Thus, it indicates that $L G_{f}=\left(L, G_{f}, f, *, \leq\right)$ is not a lattice-group under the influence of $f($.), where $f($.) is monotonically decreasing.

The determination of homeomorphism in between two lattice-groups is important in order to determine similarities in structures. However, the lattice property inherent to the lattice-groups and the multilevel mappings between spaces having different dimensions lead to difficulty in determining homeomorphism between lattice-groups. The next theorem illustrates that, the pair of mappings in two different dimensions helps in determining the homeomorphism between two lattice-groups.

\section{Theorem 10}

Let $L G_{f 1}=\left(L_{1}, G_{f 1}, f_{1}, *, \leq\right)$ and $L G_{f 2}=\left(L_{2}, G_{f 2}, f_{2}, \diamond, \leq\right)$ be two lattice-groups. If $g: L G_{f 1} \rightarrow L G_{f 2}$ is a bijection and $h: f_{1}\left(X^{2}\right) \rightarrow f_{2}\left(X^{2}\right)$, then the pair of mappings $<g, h>$ is a homeomorphism between $L G_{f 1}$ and $L G_{f 2}$ iff $h($.) is a bijection and group homeomorphism.

\section{Proof}

Let $X$ be a point set and $L G_{f 1}=\left(L_{1}, G_{f 1}, f_{1}, *, \leq\right)$ and $L G_{f 2}=\left(L_{2}, G_{f 2}, f_{2}, \diamond, \leq\right)$ be two lattice-groups, where $*: X^{2} \rightarrow X$ and $\diamond: X^{2} \rightarrow X$ are abstract algebraic operations and $\leq$ is a partial order relation in Cartesian product space.

Let in the algebraic structure be, $\forall\left\{\left(x_{a}, x_{b}\right)\right.$, $\left.\left(x_{c}, x_{d}\right)\right\} \subset X^{2}, \exists x_{\alpha}, x_{\beta}, x_{\eta}, x_{\theta} \in X$ such that:

$$
\begin{aligned}
& f_{1}\left(\left(x_{a}, x_{b}\right)\right)=x_{\alpha}, f_{1}\left(\left(x_{c}, x_{d}\right)\right)=x_{\beta}, \\
& f_{2}\left(\left(x_{a}, x_{b}\right)\right)=x_{\eta}, f_{2}\left(\left(x_{c}, x_{d}\right)\right)=x_{\theta} .
\end{aligned}
$$

As $G_{f 1}$ and $G_{f 2}$ are groups in $1 \mathrm{D}$, hence if $h: f_{1}\left(X^{2}\right) \rightarrow f_{2}\left(X^{2}\right) \quad$ is a bijection and group homeomorphism such that, $f_{1}\left(X^{2}\right) \cap f_{2}\left(X^{2}\right)=\phi$ then in this case, $h\left(x_{\alpha} * x_{\beta}\right)=h\left(x_{\alpha}\right) \diamond h\left(x_{\beta}\right)$. It indicates that:

$$
\begin{aligned}
& h\left(f_{1}\left(\left(x_{a}, x_{b}\right)\right) * f_{1}\left(\left(x_{c}, x_{d}\right)\right)\right) \\
& =h\left(f_{1}\left(\left(x_{a}, x_{b}\right)\right)\right) \diamond h\left(f_{1}\left(\left(x_{c}, x_{d}\right)\right)\right) .
\end{aligned}
$$

Moreover, let $g: L G_{f 1} \rightarrow L G_{f 2}$ be a bijection such that:

$$
\left(f_{2} \circ g\right)\left(\left(x_{a}, x_{b}\right)\right)=x_{\eta},\left(f_{2} \circ g\right)\left(\left(x_{c}, x_{d}\right)\right)=x_{\theta},
$$


where, ${ }^{\circ}$ is the composition operation.

As $h: f_{1}\left(X^{2}\right) \rightarrow f_{2}\left(X^{2}\right)$ is a bijection hence, $\exists h^{-1}$ such that, $h^{-1}\left(x_{\eta} \diamond x_{\theta}\right)=h^{-1}\left(x_{\eta}\right) * h^{-1}\left(x_{\theta}\right)$.

Moreover, if $\left(x_{a}, x_{b}\right) \leq\left(x_{c}, x_{d}\right)$ then $f_{1}\left(\left(x_{a}, x_{b}\right)\right)$ $\leq f_{1}\left(\left(x_{c}, x_{d}\right)\right)$ and, $g\left(\left(x_{a}, x_{b}\right)\right) \leq g\left(\left(x_{c}, x_{d}\right)\right)$. Furthermore, $\left(f_{2} \circ g\right)\left(\left(x_{a}, x_{b}\right)\right) \leq\left(f_{2} \circ g\right)\left(\left(x_{c}, x_{d}\right)\right) \quad$ maintaining latticegroup properties of both $L G_{f 1}$ and $L G_{f 2}$. Hence, according to the lattice-group definition, $\forall x_{\eta}, x_{\theta} \in g\left(X^{2}\right), x_{\eta} \wedge x_{\theta}=x_{\eta}, x_{\eta} \vee x_{\theta}=x_{\theta} \quad$ because, both $L G_{f 1}$ and $L G_{f 2}$ are lattice-groups.

Thus, if we denote $\langle g, h\rangle$ as a pair of mappings between the structures, $L G_{f 1}$ and $L G_{f 2}$ satisfying all the above mentioned properties in the structures, then $<g, h>$ represents the homeomorphism between two lattice-groups in Cartesian product space.

\section{Remark}

We show that, the homeomorphism $\langle g, h\rangle$ between two lattice-groups can also be formulated in a more uniform expression without involving $\exists h^{-1}$ between the two spaces. However, we derive such expression assuming a conditional homeomorphic mapping between two spaces.

Let $L G_{f 1}=\left(L_{1}, G_{f 1}, f_{1}, *, \leq\right)$ and $L G_{f 2}=\left(L_{2}, G_{f 2}, f_{2}, \diamond, \leq\right)$ be two lattice-groups and $*: X^{2} \rightarrow X$ and $\diamond: X^{2} \rightarrow X$ are abstract algebraic operations and $\leq$ is a partial order relation in Cartesian product space. Let $x_{1}, x_{2}, x_{3}, x_{4} \in X$ be such that:

$$
\begin{aligned}
& \forall\left\{\left(x_{a}, x_{b}\right),\left(x_{c}, x_{d}\right)\right\} \subset X^{2}, \\
& f_{1}\left(\left(x_{a}, x_{b}\right)\right)=x_{1}, f_{1}\left(\left(x_{c}, x_{d}\right)\right)=x_{2}, \\
& f_{2}\left(\left(x_{a}, x_{b}\right)\right)=x_{3}, f_{2}\left(\left(x_{c}, x_{d}\right)\right)=x_{4} .
\end{aligned}
$$

If $h: f_{1}\left(X^{2}\right) \rightarrow f_{2}\left(X^{2}\right)$ is a bijection and group homeomorphism such that, $f_{1}\left(X^{2}\right) \cap f_{2}\left(X^{2}\right)=\phi$ then $h\left(x_{1} * x_{2}\right)=h\left(x_{1}\right) \diamond h\left(x_{2}\right)$ because $G_{f 1}$ and $G_{f 2}$ are groups in 1D. Furthermore, let $g: L G_{f 1} \rightarrow L G_{f 2}$ be a bijection such that:

$$
\left(f_{2} \circ g\right)\left(\left(x_{a}, x_{b}\right)\right)=x_{3},\left(f_{2} \circ g\right)\left(\left(x_{c}, x_{d}\right)\right)=x_{4} \text {. }
$$

If $\exists x_{\partial} \in f_{2}\left(X^{2}\right)$ such that:

$$
\left(f_{2} \circ g\right)\left(\left(x_{a}, x_{b}\right)\right) \diamond\left(f_{2} \circ g\right)\left(\left(x_{c}, x_{d}\right)\right)=x_{\partial}
$$

then:

$$
h\left(x_{1} * x_{2}\right)=\left(f_{2} \circ g\right)\left(\left(x_{a}, x_{b}\right)\right) \diamond\left(f_{2} \circ g\right)\left(\left(x_{c}, x_{d}\right)\right)
$$

iff $h\left(x_{1} * x_{2}\right)=x_{\partial}$ indicating $x_{3} \diamond x_{4}=x_{\partial}$ in the latticegroup structure $L G_{f 2}$, where $h\left(x_{1}\right)=x_{3}$ and, $h\left(x_{2}\right)=x_{4}$.

This leads to the following conclusion:

$$
\begin{aligned}
& h\left(f_{1}\left(\left(x_{a}, x_{b}\right)\right) * f_{1}\left(\left(x_{c}, x_{d}\right)\right)\right) \\
& =\left(f_{2} \circ g\right)\left(\left(x_{a}, x_{b}\right)\right) \diamond\left(f_{2} \circ g\right)\left(\left(x_{c}, x_{d}\right)\right) .
\end{aligned}
$$

Moreover, as $\leq$ is a partial order relation maintaining lattice-group properties of both $L G_{f 1}$ and $L G_{f 2}$, hence $\forall x_{3}, x_{4} \in g\left(X^{2}\right)$, it satisfies lattice-groups properties, $x_{3} \wedge x_{4}=x_{3}, x_{3} \vee x_{4}=x_{4}$. Thus, in this case $<g, h>$ is a homeomorphism between two lattice-groups in Cartesian product space.

\section{Numerical Examples}

In this section, a set of numerical examples are given to illustrate the construction of lattices and their orientations in spaces. Moreover, the formations of lattice-monoid and lattice-group structures are presented through numerical computational examples as well as associated graph representations following the proposed algebraic structures.

\section{Oriented Lattice in $\left(\mathfrak{R}^{+}\right)^{2}$}

Let $\{(1,2),(2,3),(3,4),(3,6),(3,9),(5,12)\} \subset X^{2}$, where $X^{2} \subset\left(\mathfrak{R}^{+}\right)^{2}$. The structure of oriented lattice in positive real Cartesian product space is given in Fig. 1 .

It is evident in Fig. 1 that, the lower bound (LB) = $\{(1,2),(2,3),(3,4)\}$ and upper bound (UB) = $\{(3,6),(3,9),(5,12)\}$. Moreover, in the lattice structure, $\inf \left(X^{2}\right)=(3,4)$ and $\sup \left(X^{2}\right)=(3,6)$.

It can be observed from the oriented lattice structure that, it is monotonically increasing in $\left(\Re^{+}\right)^{2}$ space.

\section{Oriented Non-Lattice in $\left(\Re^{+}\right)^{2}$}

In this section, we present an example of nonlattice in positive real Cartesian product space. Let $\{(2,4),(4,3),(6,4),(4,4)\} \subset X^{2}$ be the set of points in $\left(\Re^{+}\right)^{2}$. The oriented but non-lattice structure is presented in Fig. 2. It is clear from Fig. 2 that, it is following the definition of the partial order relation $\leq$ because, every pair of lattice elements satisfies following required condition:

$$
\left[\left(x_{a}, x_{b}\right) \leq\left(x_{c}, x_{d}\right)\right] \Rightarrow\left[\left(x_{a} \leq x_{c}\right) \wedge\left(x_{b} \leq x_{d}\right)\right] .
$$

However, in this case, it is not a lattice in $2 \mathrm{D}$, since the first pair of elements does not satisfy relation $\leq$ as:

$$
[(4,3) \leq(2,4)] \Rightarrow \neg[(4 \leq 2) \wedge(3 \leq 4)]
$$


A lattice in Cartesian product space (positive reals)

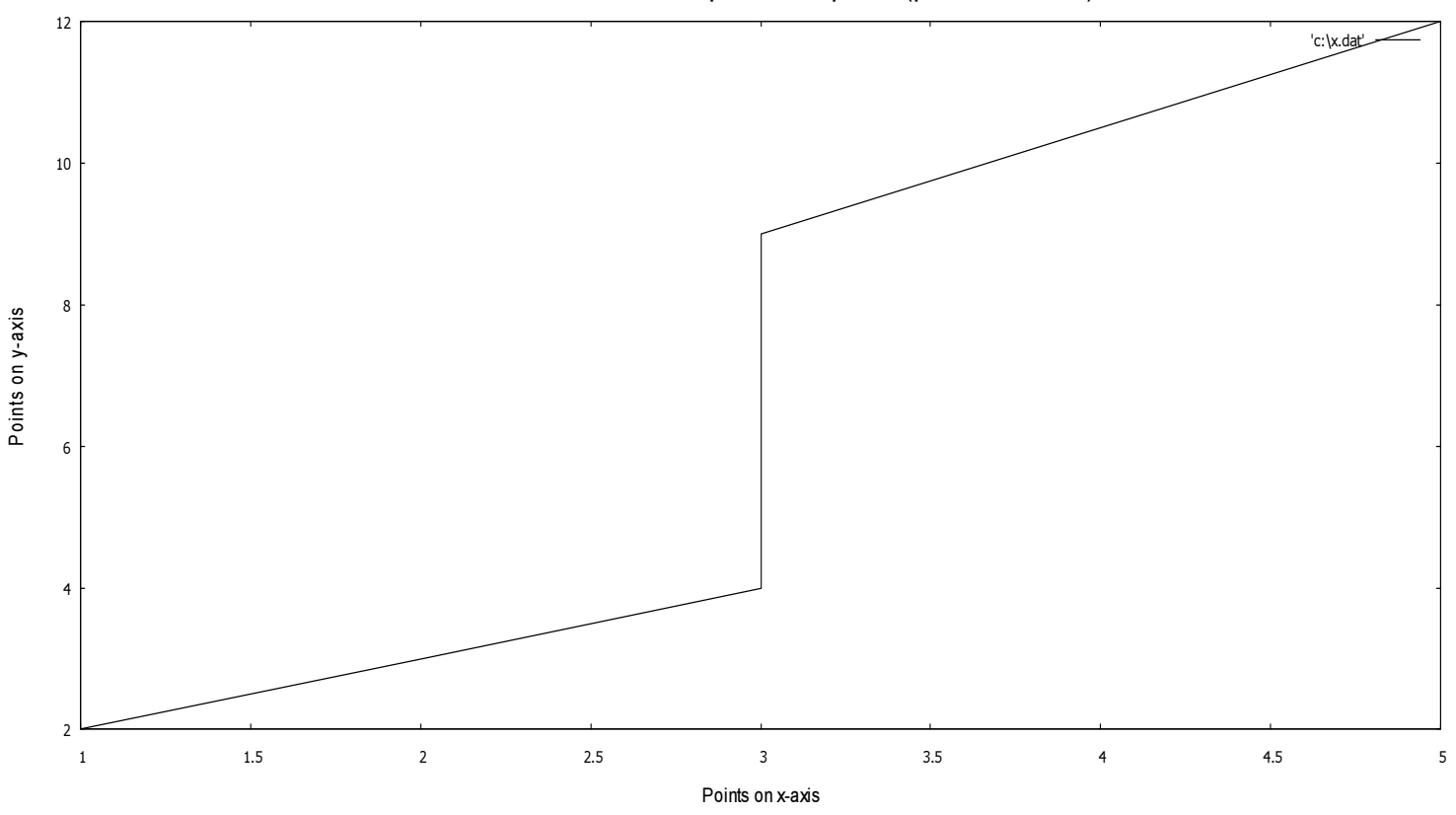

Fig. 1: An oriented lattice representation in real Cartesian product space (strictly positive)

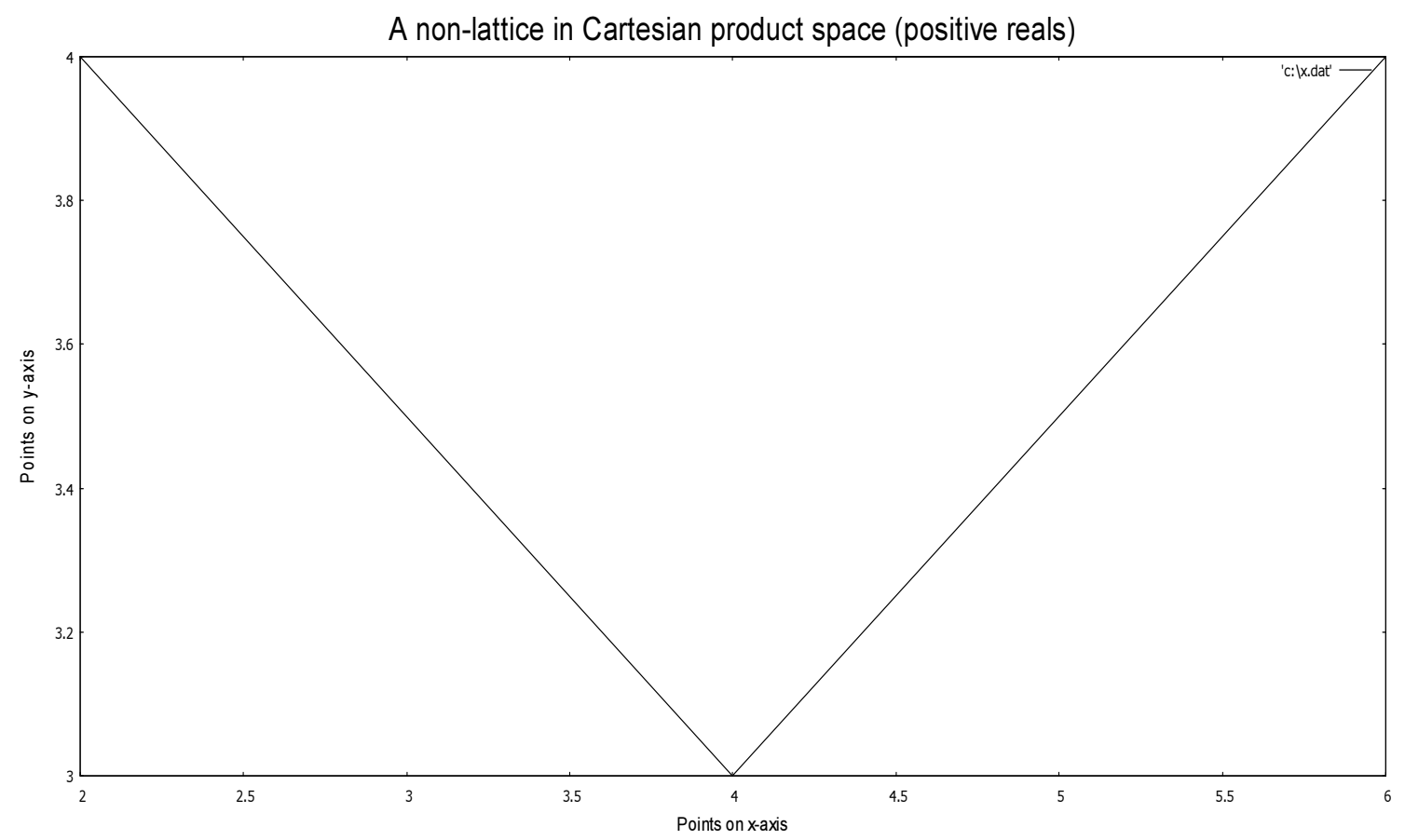

Fig. 2: Example of non-lattice representation in real Cartesian product space (strictly positive)

\section{Oriented Partially Ordered Monoid in $\mathfrak{R}^{2}$}

In this section, we present an example of partially ordered monoid in real Cartesian product space.

Let $\{(-9,-5),(-4,-3),(-2,4),(0,0)\}$ be the basis set in Cartesian product space (a subset of $\mathfrak{R}^{2}$ ) having partial ordering relation, where the monoid operation is $*=+$. Evidently, it satisfies the following latticemonoid property as:

$$
\begin{aligned}
& {[(-9,-5) \leq(-4,-3)] \Rightarrow\left[(-9,-5)+{ }_{2}(-2,4)\right.} \\
& \left.\leq(-4,-3)+{ }_{2}(-2,4)\right] .
\end{aligned}
$$




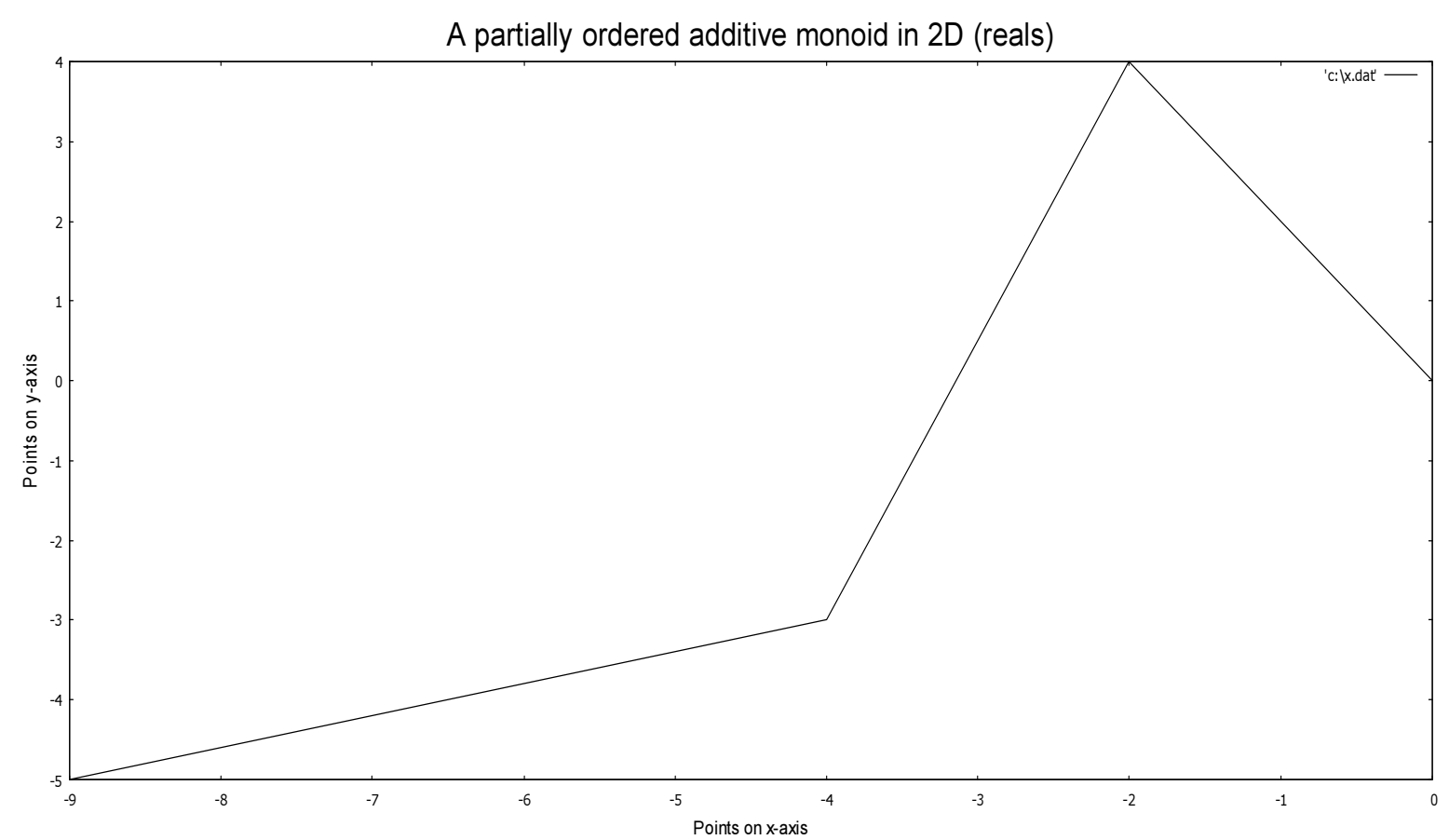

Fig. 3: A partially ordered monoid in Cartesian product space (additive)

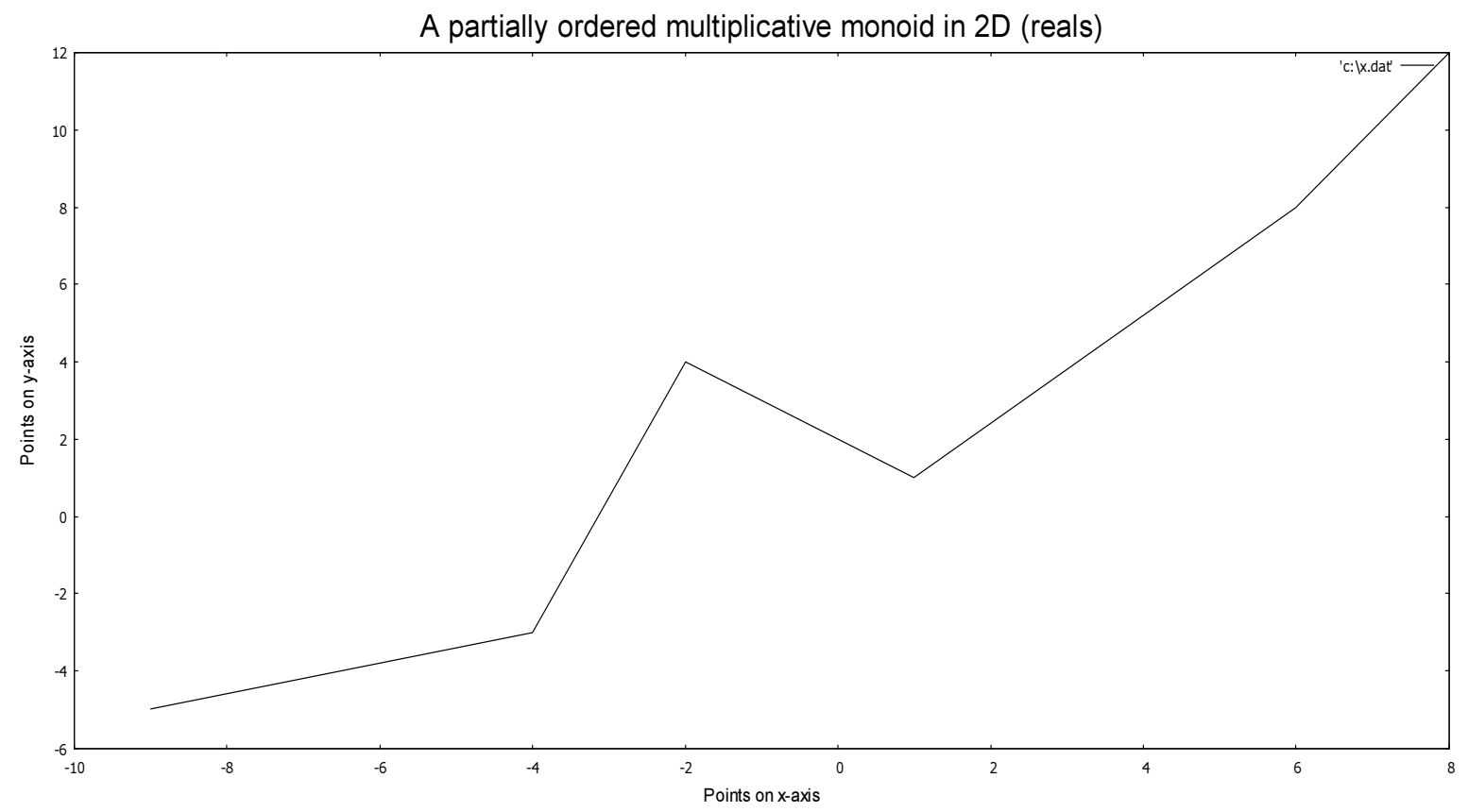

Fig. 4: Partially ordered monoid representation in Cartesian product space (multiplicative)

The representation of resulting partially ordered monoid is given in Fig. 3 .

Again, if we select the basis set as $\{(-9,-5),(-4,-3)$, $(-2,4),(6,8),(8,12),(1,1)\}$ and we change the algebraic operation as $* \equiv \cdot$ (multiplicative), then the resulting partially ordered monoid is presented in Fig. 4.

\section{Not a Partially Ordered Monoid in $\mathfrak{R}^{2}$}

The representation of not a partially ordered monoid in $2 \mathrm{D}$ is given in Fig. 5, where $\{(2,3),(4,5),(6,12)$, $9,12)\} \subset N^{2} \subset \mathfrak{R}^{2}$ and $* \equiv \cdot$ (multiplicative). We have considered $\leq$ as the poset relation. 


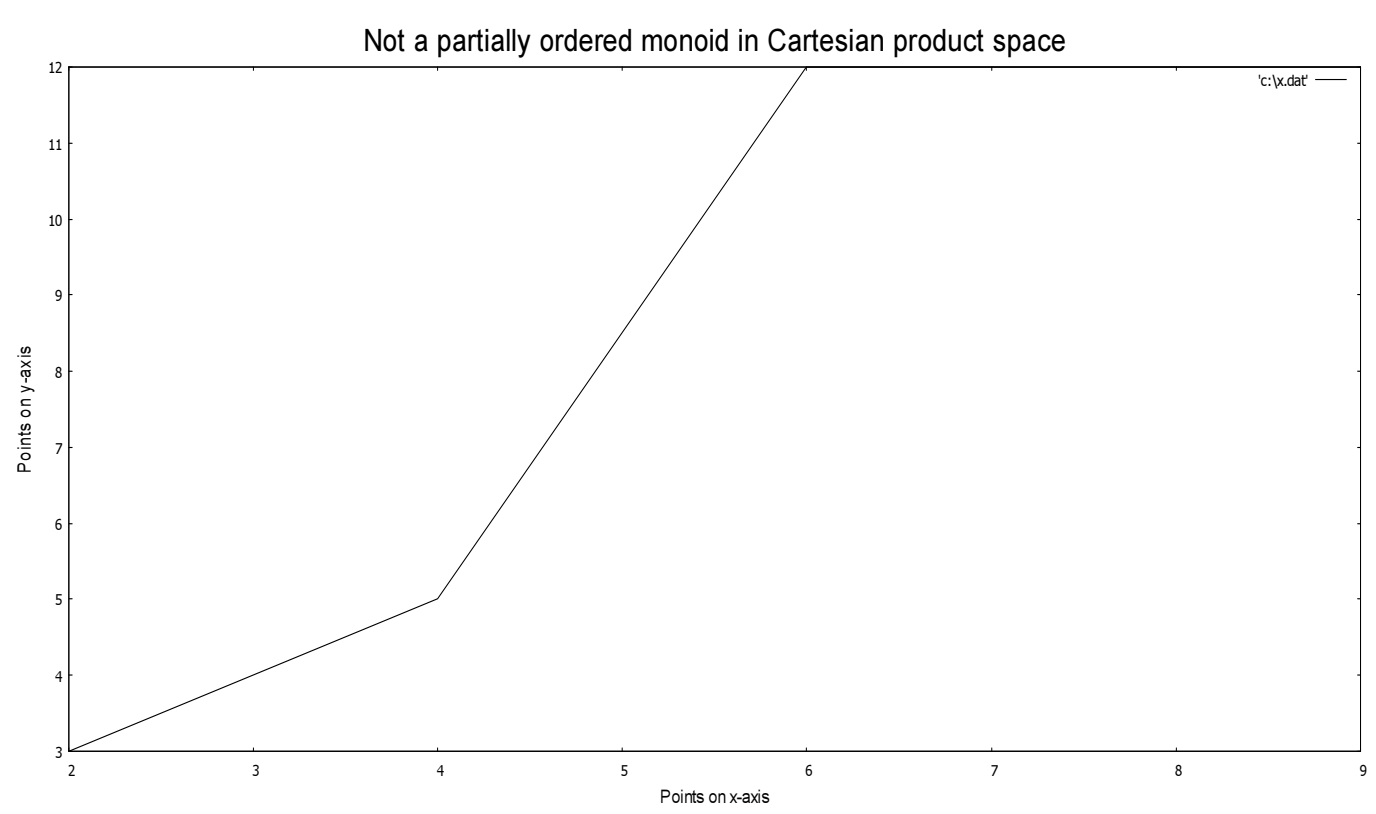

Fig. 5: Not a partially ordered monoid in real Cartesian product space

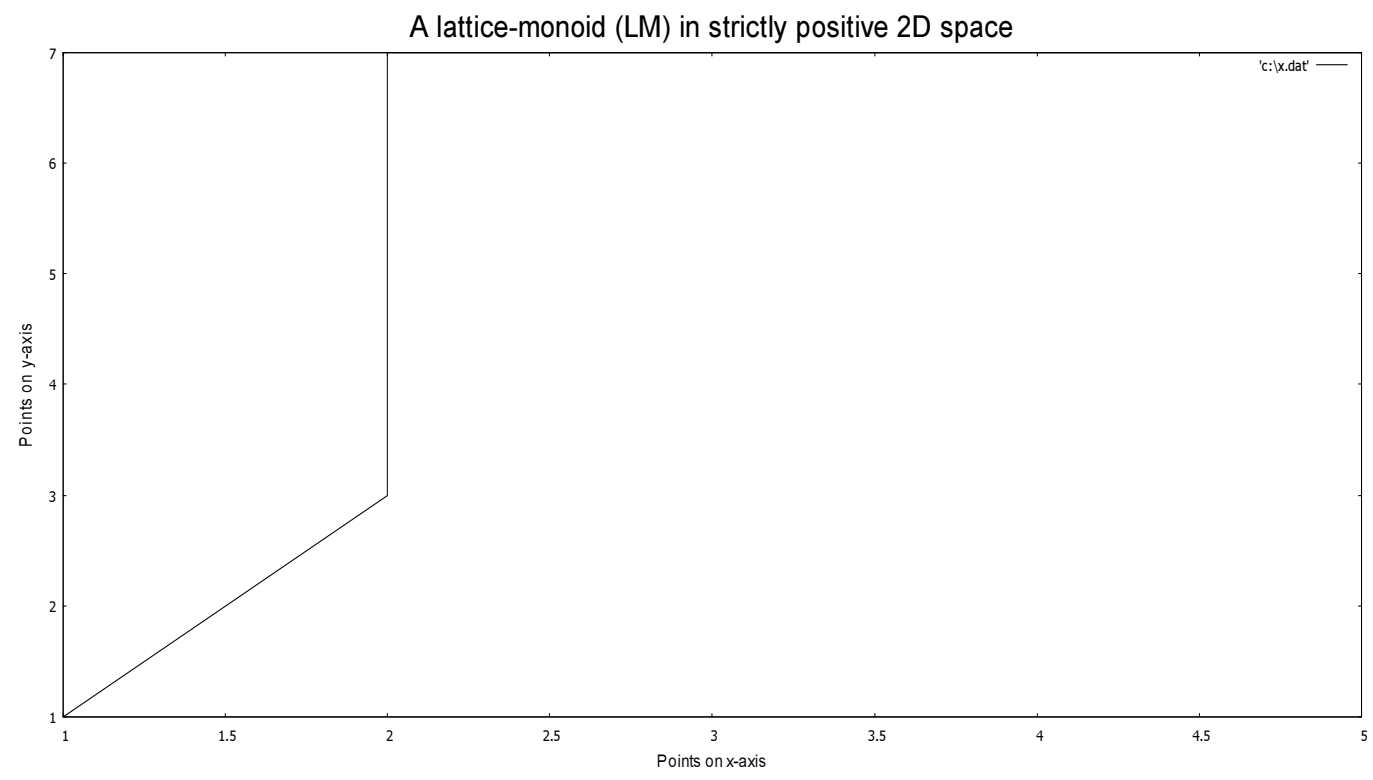

Fig. 6: A lattice-monoid representation in real Cartesian product space (strictly positive)

Following the definition of partially ordered monoid, $(2,3),(4,5) \in \mathrm{N}^{2} \subset \mathfrak{R}^{2}$ and, $(-1,-2) \in \mathrm{X}^{2} \subset \mathfrak{R}^{2}$. The following computation illustrates the reasoning:

$$
[(2,3) \leq(4,5)] \equiv \neg\left[\left((2,3) \cdot \cdot_{2}(-1,-2)\right) \leq\left((4,5) \cdot_{2}(-1,-2)\right)\right] .
$$

Hence, one can conclude that,
$[(2,3) \leq(4,5)] \neq \neg[(-2,-6) \leq(-4,-10)]$. Thus, the set given by, $\{(2,3),(4,5),(6,12),(9,12)\} \subset \mathrm{N}^{2} \subset \mathfrak{R}^{2}$ does not satisfy the definition of partially ordered monoid.

\section{A Lattice-Monoid in $2 D$}

In this section, we present an example of lattice-monoid structure in 2D. Let $\{(1,1),(2,3),(2,7),(5,7)\}$ be the basis set in Cartesian product space, where $* \equiv \cdot$ (multiplicative). Evidently, the following lattice property is satisfied by it:

$$
\begin{aligned}
& (1,1) \cdot \cdot_{2}[(2,3) \wedge(2,7)]=\left[(1,1) \cdot \cdot_{2}(2,3)\right] \wedge \\
& {\left[(1,1) \cdot \cdot_{2}(2,7)\right],(1,1) \cdot \cdot_{2}(2,3)=(2,3), \text { and, }} \\
& (1,1) \cdot \cdot_{2}[(2,3) \vee(2,7)]=\left[(1,1) \cdot \cdot_{2}(2,3)\right] \\
& \vee\left[(1,1) \cdot \cdot_{2}(2,7)\right],(1,1) \cdot \cdot_{2}(2,7)=(2,7) .
\end{aligned}
$$

Figure 6 presents the lattice-monoid in real Cartesian product space under multiplication.

It can be observed in Fig. 6 that, the lattice-monoid structure in 2-dimensional positive real surface is 
oriented in nature. Moreover, the oriented structure is not a monotonic increase or decrease as a chain on the positive real surface.

\section{Not a Lattice-Monoid in $2 D$}

In this section, we present a counter example illustrating a non-lattice-monoid in $2 \mathrm{D}$ space.

Let $\{(1,1),(1,2),(2,4),(3,9),(4,5)\} \subset \mathrm{X}^{2}$ be in Cartesian product space. Figure 7 represents the example of nonlattice-monoid structure. The reason is that, in 2D, $\{(3,9),(4,5)\} \not \subset \leq$.

\section{Lattice-Group in Cartesian Product Space}

In this section, we present an example illustrating lattice-group in Cartesian product space. We start with a simple coset of elements to illustrate the basic structure of lattice-group. We consider that $L G_{f}=\left(L, G_{f}, f,+, \leq\right)$ is a lattice- group, where $L$ is a lattice and $G_{f}=(M,+, f)$ is an additive group in $X$. Moreover, we consider surjective function defined as, $f((a, b))=a+b$.

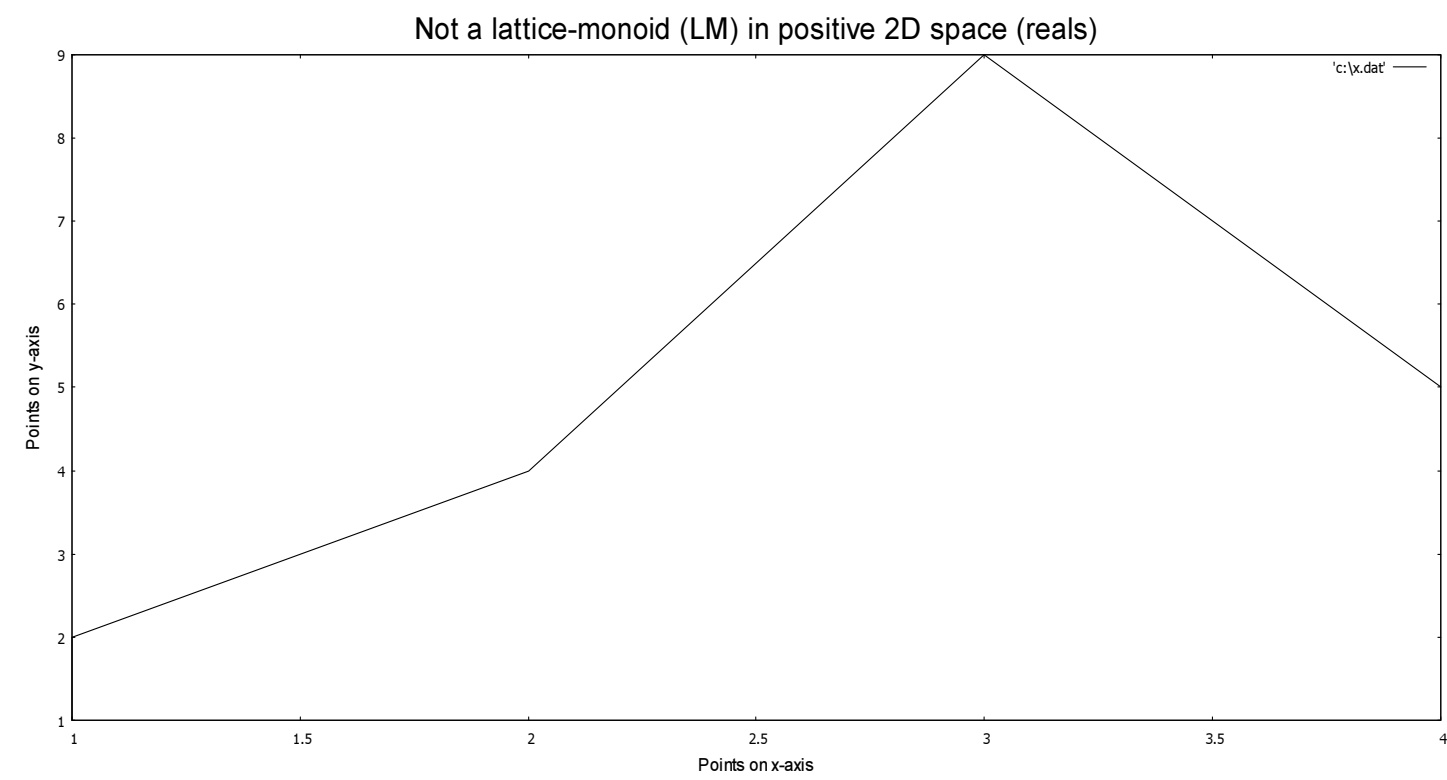

Fig. 7: A non-lattice-monoid representation in real Cartesian product space (strictly positive)

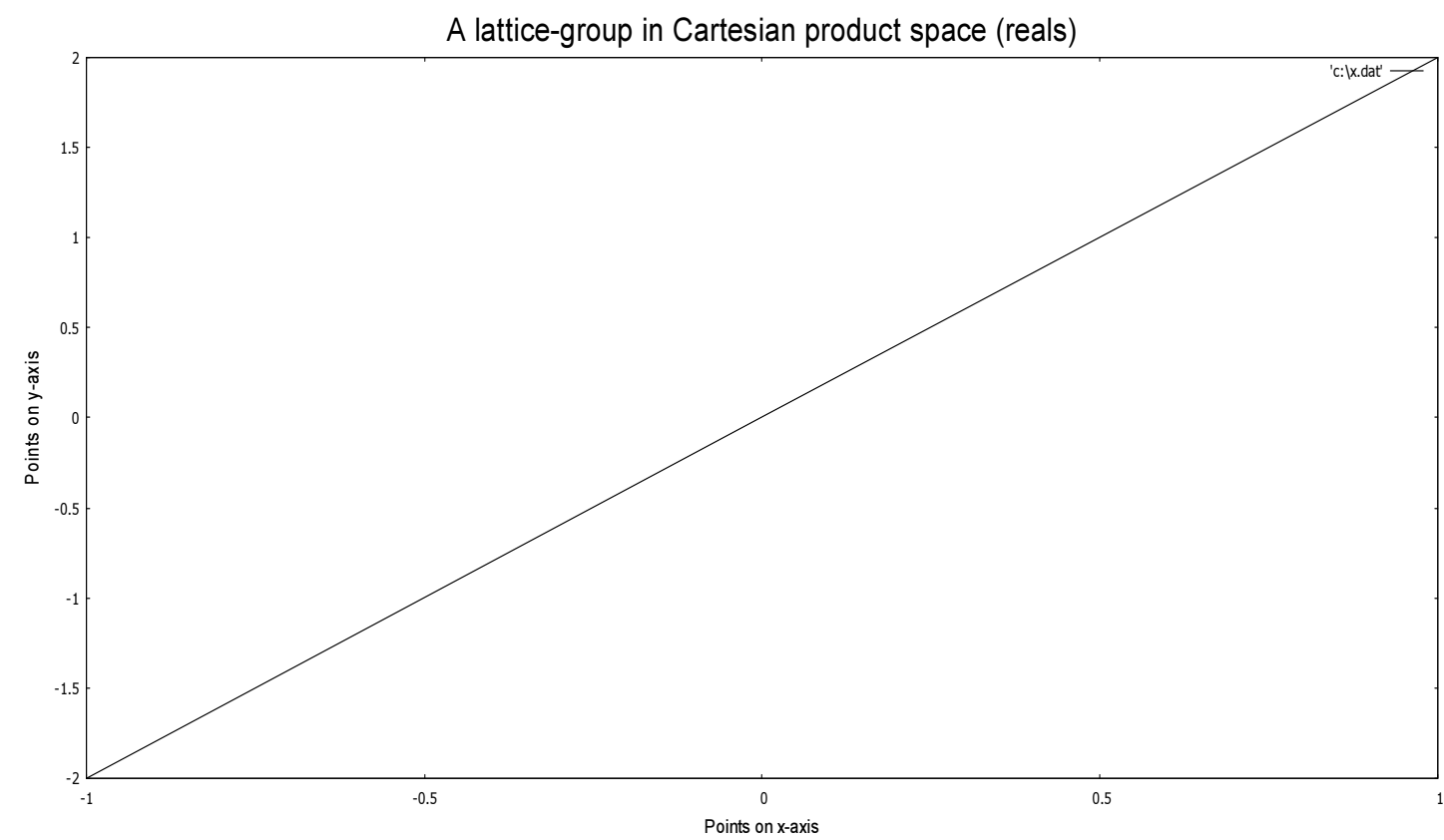

Fig. 8: An additive lattice-group representation in Cartesian product space 
Let $\{(-1,-2),(0,0),(1,2)\} \subset \mathfrak{R}^{2}$ be in $2 \mathrm{D}$ reals, where $* \equiv+$ and $, \quad f((-1,-2))=-3, f((0,0))=0, f((1,2))=3$ and, according to the lattice-group definition, $-3 \wedge 0=-3,-3 \vee 0=0$.

Figure 8 illustrates the example of additive latticegroup structure. Furthermore, in a more generalized form, if $X \subseteq Z$ then there exists at least one surjective $\mathrm{f}$ : $X^{2} \rightarrow X$ such that, $L G_{f}=\left(Z, G_{f}, f,+, \leq\right)$ is a lattice- group, where $Z$ is a lattice-chain (integers) and $G_{f}=\left(X^{2},+, f\right)$ is an additive group in $X$.

\section{Comparative Evaluation}

In this section, we have presented the comparative analysis of properties of lattice-group structure with the other contemporary groups in the domain. The comparative analysis is based on a set of parameters such as, finiteness, compactness, commutativity, symmetry, cyclicness, convexity, direction and orientation. First, we present a set of properties of various group structures, which is summarized in Table 1.

The lateral completion of an arbitrary lattice group incorporates finite and Abelian as well as symmetry properties employing function (Bernau, 1975). Moreover, it can be cyclic depending on the function where it considers orientation and convexity. The partially ordered group is finite and Abelian where it is symmetric and it can be convex and directed in nature (Shirshova, 2015). The dihedral group $\left(D_{n}\right)$ considers symmetry group structure, which is finite in nature. The Abelian and cyclic properties of dihedral group is dependent on group order. A dihedral group is Abelian as well as cyclic if the group order is in $\{1,2\}$ (Bilal et al., 2013). Interestingly, if the group order $\mathrm{n} \geq 3$, then $\left(D_{n}\right)$ becomes a non-Abelian acyclic group. However, the Abelian and cyclic property of proposed lattice-group in this paper independent of group order. In the lattice-group, the group order is determined by the type of function mapping between spaces having different dimensions. There are different varieties of groups considering lattice structures. For example, the wallpaper group is a lattice based structure, which is finite and symmetric (Schattschneider, 1978). The finiteness and symmetry of wallpaper group are maintained based on two dimensional repetitive patterns preserving symmetries. Moreover, the wallpaper group is Abelian and cyclic in nature (Schattschneider, 1978). On the other hand, the proposed lattice-group constructs an Abelian structure under any arbitrary function (i.e., surjective, injective and bijective). The proposed lattice-group can be constructed as a cyclic group if and only if the function is surjective and homeomorphic. The alternating group $\left(A_{n}\right)$ is a group variety based on even permutations and preserves finite and symmetry properties (Donold, 1968). An alternating group can be Abelian if and only if the group order is $n \leq 3$. The Z3 type of alternating group is cyclic in nature (Donold, 1968). However, the proposed lattice-group is a cyclic group under the influence of surjective functions. The simple Lie group is a non-Abelian group structure and it is finite as well as symmetric (Milne, 2013). The embedding of topological lattice-ordered group are locally compact, where the group is Abelian (Robert, 1969). In this group, the cyclicness and symmetry depend of function mapping between spaces. If the function is surjective then there exists a relation-center in the lattice-group. However, if the function is odd then the lattice-group does not exist under addition in Cartesian product space. Furthermore, if the odd function and even function are surjective as well as homeomorphic under multiplication then the lattice-group cannot be constructed. Interestingly, if the monotonically decreasing function mapping between spaces is applied, then the lattice-group cannot exist in the Cartesian product space. The proposed lattice-group under the influence of function proposed in this paper is finite. Finally, the lattice-groups exhibit the group homeomorphism under the influence of a pair of mappings between spaces having varying dimensions.

Table 1: Comparison of various properties of different group structures ( $\mathrm{n}$ is group order)

\begin{tabular}{|c|c|c|c|c|c|c|}
\hline Group types & $\begin{array}{l}\text { Finiteness/ } \\
\text { compactness }\end{array}$ & Commutativity & Symmetry & Cyclicness & Convexity & Direction/orientation \\
\hline $\begin{array}{l}\text { The lateral completion of } \\
\text { an arbitrary lattice group }\end{array}$ & Finite & Abelian & $\begin{array}{l}\text { Depends on } \\
\text { function }\end{array}$ & $\begin{array}{l}\text { Depends on } \\
\text { function }\end{array}$ & Convexity & Oriented \\
\hline Partially ordered group & Finite & Abelian & Yes & No & Convexity & Directed \\
\hline Dihedral group & Finite & Abelian (if $\mathrm{n}=1,2$ ) & Yes & Yes (if $n=1,2$ ) & Convexity & $\begin{array}{l}\text { Oriented in the three } \\
\text { dimensional case }\end{array}$ \\
\hline Wallpaper group & Finite & Abelian & Yes & Yes & Convexity & Directed \\
\hline Alternating group & Finite & Abelian (if $\mathrm{n} \leq 3$ ) & Yes & $\begin{array}{l}\text { Yes (if only } \\
\text { Z3 type) }\end{array}$ & Convexity & Directed/oriented \\
\hline Simple lie group & Finite & Non-Abelian & Yes & No & Convexity & Oriented \\
\hline $\begin{array}{l}\text { Embeddings of topological } \\
\text { lattice-ordered group }\end{array}$ & $\begin{array}{l}\text { Locally } \\
\text { compact }\end{array}$ & Abelian & $\begin{array}{l}\text { Depends on } \\
\text { function }\end{array}$ & $\begin{array}{l}\text { Depends on } \\
\text { function }\end{array}$ & Convexity & Oriented \\
\hline Littice-group & Finite & Abelian & $\begin{array}{l}\text { Depends on } \\
\text { function }\end{array}$ & $\begin{array}{l}\text { Depends on } \\
\text { function }\end{array}$ & Convexity & Oriented \\
\hline
\end{tabular}




\section{Conclusion}

In this paper, we have constructed the algebraic structures hybridizing lattice, monoid and group employing various mappings under certain conditions. First, we prepare the monoid structure in Cartesian product space by using an arbitrary algebraic operation in space having closure property. In order to generalize the concept, the inverses of points in Cartesian product space are not considered to be same as the ordered pairs of respective individual inverses in the space. We illustrate that, the monoid in Cartesian product space can retain the poset relation successfully generating the partially ordered monoid. Before proceeding to the lattice-group, the generalized $2 \mathrm{D}$ lattice structure is prepared and hybridized with the monoid to establish the lattice-monoid in Cartesian product space. This paper illustrates that there exists lattice-group structure under the influence of surjective functions. Moreover, the extension of surjective function into surjectivehomeomorphic function generates cyclic group. Furthermore, the distributive lattice-subgroup can exist under specific condition and, in such case the mapping function can be arbitrary in nature. Interestingly, the monotonically decreasing function does not support existence of a lattice-group. If the group is Abelian and is prepared employing surjective function, then a relation center exists in the lattice-group. The relation center is formulated by applying constructs of relational algebra including inverse relations. Interestingly, the relation centers exist in both additive and multiplicative latticegroups. The construction and analysis of group homeomorphisms have resulted in the formulation of a function pair to establish the homeomorphism between two lattice-groups. The presented numerical examples illustrate geometric representations of various structures. A set of counter examples are also presented in numerical forms. The comparative analysis explains a set of distinctive characteristics of the proposed latticegroup in the family of other group varieties.

\section{Acknowledgement}

Authors would like to thank Editors and anonymous reviewers for valuable comments.

\section{Funding Information}

This work is partly funded by Gyeongsang National University, Jinju, ROK.

\section{Author's Contributions}

First author (D. Malikov) proposed the concept, proposed theorems, proofs and drafted the paper. Second and corresponding author (S. Bagchi) refined the concept, refined theorems and, corrected the draft.

\section{Ethics}

Authors declare that there are no ethical issues in the paper.

\section{References}

Attiya, H., M. Herlihy and O. Rachman, 1995. Atomic snapshots using lattice agreement. Distributed Comput., 8: 121-132.

Bilal, N.A., A.A. Othman and S.A. Mousa, 2013. Dihedral groups of order $2^{\mathrm{m}+1}$. Int. J. Applied Math., 26: 1-7.

Birkhoff, G., 1967. Lattice theory. American mathematical society.

Bernd, S., 2016. Ordered Sets: An Introduction with Connections from Combinatorics to Topology. 2rd Edn., Birkhauser, Basel, ISBN-10: 3319297880, pp: 420.

Bernau, S.J., 1975. The lateral completion of an arbitrary lattice group. J. Australian Math. Society, 19: 263-289.

Chase, C.M. and V.K. Garg, 1995. Efficient detection of restricted classes of predicates. Proceedings of the International Workshop on Distributed Algorithms, (WDA' 95), France, Springer, pp: 303-317.

DOI: $10.1007 / \mathrm{BFb} 0022155$

Dushnik, B. and E.W. Miller, 1941. Partially ordered sets. Am. J. Math., 63: 600-610.

Donold, S.P., 1968. Permutation Groups. 1st Edn., Benjamin Company, New York, ISBN-13: 9780486485928.

Frank, J.O., 2000. An application of lattice theory to knowledge representation. Theoretical Comput. Sci., 249: 163-196. DOI: 10.1016/S0304-3975(00)00058-X

George, G., 2009. Lattice Theory: First Concepts and Distributive Lattices. 1st Edn., Dover Publications, USA, ISBN-10: 048647173X, pp: 240.

Herstein, I.N., 1975. Topics in Algebra. 1st Edn., John Wiley Sons, USA, ISBN-10: 0471010901, pp: 388.

Lamport, L., 1978. Time, clocks and the ordering of events in a distributed system. Commun. ACM, 21: 558-565.

Louis, N., 2016. An Introduction to Lattice based probability theories. J. Math. Psychol., 74: 66-81. DOI: 10.1016/j.jmp.2016.04.013

Laszlo, F., 2015. Abelian Groups. 1st Edn., Springer, Cham, ISBN-10: 3319194224, pp: 747.

Milne, J.S., 2013. Lie Algebras. Algebraic Groups and Lie Groups Version 2.00.

Vasco, M.I.G. and R. Steinwandt, 2015. Group Theoretic Cryptography. 1st Edn., Chapman Hall/CRC, Boca Raton, ISBN-10: 1584888377, pp: 244.

Mildred, S.D., D. Gene and J. Ado, 2010. Group theory Applications of Physics of Condensed Matter. Springer, Berlin, ISBN-10: 3642069452, pp: 582. 
Mauricio, O., J. Bharat and A.P. David, 1999. Theory of partial-order programming. Sci. Comput. Programm., 34: 207-238.

Robert, L.M., 1969. Embeddings of topological latticeordered groups. Trans. Am. Math. Society, 146: 447-455. DOI: $10.2307 / 1995184$

Seymour, L. and L. Marc, 2007. Schaum's Outline of Discrete Mathematics. 3rd Edn., The McGraw-Hill Companies, ISBN-10: 0071511016, pp: 474.

Scott, W.R., 1987. Group Theory. 1st Edn., Dover Publications INC, ISBN-10: 0486653773.

Shirshova, E.E., 2015. On values of elements in partially ordered group. J. Math. Sci., 206: 597-607.

Sen, A. and V.K. Garg, 2003. Partial Order Trace Analyzer (POTA) for distributed programs. Electronic Notes Theoretical Comput. Sci., 89: 22-43. DOI: $10.1016 / \mathrm{S} 1571-0661(04) 81041-7$

Schattschneider, D., 1978. The plane symmetry groups. Am. Math. Monthly, 85: 439-450.

Thomas, D., 2004. Lattice Theory. 1st Edn., Elsevier, London, ISBN-10: 1483147495, pp: 296.
Tzu-Chun, L., 2018. A study of non-abelian public key cryptography. Int. J. Netw. Security, 20: 278-290.

Vijay, K.G., 2015. Introduction to Lattice Theory with Computer Science Applications. 1st Edn., Wiley, New Jersey, ISBN-10: 1118914376, pp: 272.

Wen-Hai, L., W.K. Liang and G. Shun-Quan, 1990. Applications of lattice theory to graph decomposition. Circuits Syst. Signal Process., 9: 181-195.

William, F.T., 2013. Introduction to Real Analysis. 1st Edn., Pearson Education, ISBN-10: 0130457868, pp: 574.

Walter, R., 1976. Principles of Mathematical Analysis. 3rd Edn., McGraw-Hill, Singapore, ISBN-10: 0070856133, pp: 342.

Xiong, Z., H. Changyong and K.G. Vijay, 2018. Lattice agreement in message passing systems. Proceedings of the 32nd International Symposium on Distributed Computing, (SDC' 18), Leibniz International Proceedings in Informatics, Germany. 